\title{
LONGITUDINAL BEAM DYNAMICS WITH RF NOISE
}

\author{
H.-J. SHIH, ${ }^{1}$ J.A. ELLISON,${ }^{2}$ B.S. NEWBERGER ${ }^{3}$ and R. COGBURN ${ }^{2}$ \\ ${ }^{1}$ Superconducting Super Collider Laboratory, ${ }^{2} 2550$ Beckleymeade Avenue, \\ Dallas, TX 75237, USA \\ ${ }^{2}$ Department of Mathematics and Statistics, The University of New Mexico, \\ Albuquerque, NM 87131, USA \\ ${ }^{3}$ Institute for Fusion Studies, The University of Texas at Austin, \\ Austin, TX 78712, USA
}

(Received 17 August 1992; in final form 26 August 1993)

The Dôme-Krinsky-Wang (DKW) diffusion-in-action theory for rf-noise-induced emittance dilution is reviewed and related to recent work on the approximation of stochastic processes by Markov processes. An accurate and efficient numerical procedure is developed to integrate the diffusion equation of the DKW theory. Tracking simulations are undertaken to check the validity of the theory in the parameter range of the Superconducting Super Collider (SSC) and to provide additional information. The study of effects of rf noise is applied to two problems of interest at the SSC: (1) determination of noise tolerance levels in the rf system, and (2) feasibility of beam extraction using crystal channeling.

KEY WORDS: Particle Dynamics, Radio-frequency Devices, Storage Rings, Synchrotrons: Proton, Longitudinal, Dynamics, RF, Noise, SSC, Crystal, Extraction

\section{INTRODUCTION}

Emittance blowup and beam loss due to intrinsic noise in the rf accelerating system has been observed in the Super Proton Synchrotron (SPS) ${ }^{1-3}$ and in other proton storage rings. It is therefore important to understand the effect of rf noise on the longitudinal dynamics of particle beams in high-energy accelerators. Dôme ${ }^{4}$ and Krinsky and Wang ${ }^{5}$ have independently provided a theoretical basis in which the effect of noise is described by a Markov diffusion process in longitudinal action. In Section 2 we review the Dôme, Krinsky, Wang (DKW) diffusion theory and relate it to modern ideas in the theory of stochastic processes. We have numerically integrated the diffusion equation that describes the evolution of the action distribution in the

\footnotetext{
* Operated by the Universities Research Association, Inc., for the U.S. Department of Energy under Contract No. DE-AC35-89ER40486.
} 
TABLE 1: General SSC Parameters.

\begin{tabular}{lll}
\hline$h$ & harmonic number & 104544 \\
$\alpha_{c}$ & momentum compaction factor & $9.1 \times 10^{-5}$ \\
$p_{s}$ & synchronous momentum, TeV/c & 20 \\
$V$ & peak rf voltage, $\mathrm{MV}$ & 20 \\
$f_{o}$ & revolution frequency, $\mathrm{Hz}$ & 3441 \\
$C_{o}$ & circumference, $\mathrm{m}$ & 87120 \\
$\Omega / 2 \pi$ & small oscillation synchrotron frequency, $\mathrm{Hz}$ & 4.23 \\
$\epsilon_{N}$ & normalized emittance, $\mathrm{m} \cdot \mathrm{rad}$ & $10^{-6}$ \\
\hline
\end{tabular}

DKW theory. Using the parameters of the Superconducting Super Collider (SSC), we have compared our numerical results with tracking simulations and found good agreement; this agreement gives us confidence in using the theory. This is discussed in Section 3, where we describe our numerical procedures and tracking simulations and give a detailed comparison. In Section 4 we apply the formalism and the associated numerical computations to two problems of interest at the SSC: the issue of the longitudinal emittance increase due to noise in components of the rf system of the SSC collider ring, and the possibility of extraction of a low-intensity proton beam for high-precision $B$ physics in a fixed-target spectrometer. Some general SSC parameters are listed in Table 1.

\section{REVIEW OF THE DKW THEORY}

The underlying theory is reviewed in this section, and readers are referred to Dôme ${ }^{4}$ for more details. For a sinusoidal rf voltage with phase and amplitude noise, the turn-to-turn energy and phase variations in a stationary bucket are given by

$$
\begin{aligned}
& \phi_{n+1}-\phi_{n}=P_{n+1}+\psi_{n+1}-\psi_{n}, \\
& P_{n+1}-P_{n}=-K_{0}\left(1+a_{n}\right) \sin \phi_{n},
\end{aligned}
$$

with $P_{n}=2 \pi h \eta\left(\Delta p_{n} / p_{s}\right), K_{0}=2 \pi h \eta\left(e V / p_{s} v_{s}\right)$, and $\eta=\alpha_{c}-1 / \gamma^{2}$. See Table 1 for $h, \alpha_{c}, p_{s}$ and $V$. Here $n$ is the turn number, $\phi$ the phase relative to the synchronous phase, $\psi$ the phase noise, $a$ the amplitude noise, $\gamma$ the ratio of the particle's total energy to its rest energy, $v_{s}$ the velocity of the synchronous particle, and $e$ the electron charge; $\Delta$ refers to a variation relative to the synchronous particle. If we denote the beam revolution period by $T_{0}$, define $P=P_{n} / T_{0}$, divide Equation (2.1a) by $T_{0}^{2}$ and Equation (2.1b) by $T_{0}$, and approximate the difference quotients by derivatives, then we obtain the continuous time approximation of Equation (2.1): 


$$
\begin{aligned}
& \dot{\phi}=P+\dot{\psi}(t), \\
& \dot{P}=-\Omega^{2}(1+a(t)) \sin \phi
\end{aligned}
$$

Here we have used $\Omega=\sqrt{K_{0}} / T_{0}$. In what follows we take $a(t)$ and $\psi(t)$ to have zero mean for each $t$. Equation (2.2) can be derived from the time-dependent Hamiltonian:

$$
H(\mathbf{x}, t)=H_{0}(\mathbf{x})+H_{1}(\mathbf{x}, t)
$$

with

$$
\begin{aligned}
H_{0}(\mathbf{x}) & =\frac{1}{2} x_{2}^{2}+U\left(x_{1}\right), \\
H_{1}(\mathbf{x}, t) & =a(t) U\left(x_{1}\right)+\dot{\psi}(t) x_{2} .
\end{aligned}
$$

Here $\mathbf{x}=\left(x_{1}, x_{2}\right)^{T}$ with $x_{1}=\phi$ and $x_{2}=P$, and $U\left(x_{1}\right)=\Omega^{2}\left(1-\cos x_{1}\right)$.

In the unperturbed case $(a=\psi=0)$, the action $J=(1 / 2 \pi) \oint P d \phi$ is conserved. In the perturbed case, Dôme assumes conditions are such that the action evolves by a Markov diffusion process and then shows that the coefficients in the Fokker-Planck equation are related, so the action density $\rho(J, t)$ evolves via the diffusion equation

$$
\frac{\partial \rho}{\partial t}=\frac{\partial}{\partial J}\left(D(J) \frac{\partial \rho}{\partial J}\right) .
$$

The natural auxiliary conditions are

$$
\begin{aligned}
\rho(J, 0) & =\rho_{0}(J), \\
\rho\left(J_{b}, t\right) & =0,
\end{aligned}
$$

where $\rho_{0}(J)$ is the initial density with $\int_{0}^{J_{b}} \rho_{0}(J) d J=1$, and the second condition is an absorbing boundary condition at $J=J_{b}$. It is reasonable to take $J_{b} \lesssim J_{s}$, where $J_{s}$ is the action at the separatrix, because once a particle comes close to the separatrix it is effectively lost due to for example, collimators employed to reduce background in the detectors. Because $D(0)=0$, no boundary condition at $J=0$ is needed.

To give the reader a feel for the connection between the phase space and action descriptions, we derive the diffusion coefficient $D(J)$. The transformation from $\mathbf{x}$ to action-angle variables $(J, \theta)$ is as follows. Given $\mathbf{x}$, the energy is

$$
h=\frac{1}{2} x_{2}^{2}+U\left(x_{1}\right)
$$


and the action

$$
J(h)=\frac{1}{2 \pi} 4 \sqrt{2} \int_{0}^{\phi_{m}} \sqrt{h-U\left(x_{1}\right)} d x_{1},
$$

where $\phi_{m}$ is the maximum value of $x_{1}$ for the orbit defined by (2.6), i.e.,

$$
U\left(\phi_{m}\right)=h .
$$

The canonically conjugate angle $\theta$ is given by

$$
\theta=\omega_{s}(J) \tau
$$

where $\tau$ is the time it takes to go from $\left(\phi_{m}, 0\right)$ to $\left(x_{1}, x_{2}\right)$ moving counterclockwise in the unperturbed problem, and

$$
\omega_{s}(J)=h^{\prime}(J),
$$

where $h(J)$ is the inverse of $J(h)$ defined in (2.7). If we let $\mathbf{x}=\mathbf{g}(\tau, \mathbf{u})$ be the general solution of the unperturbed problem, i.e.,

$$
\begin{aligned}
\frac{\partial \mathbf{g}(\tau, \mathbf{u})}{\partial \tau} & =\left(\begin{array}{cc}
0 & 1 \\
-1 & 0
\end{array}\right) \nabla H_{0}(\mathbf{g}(\tau, \mathbf{u})), \\
\mathbf{g}(0, \mathbf{u}) & =\mathbf{u},
\end{aligned}
$$

where $\nabla H_{0}(\mathbf{x})=\left(\frac{\partial H_{0}}{\partial x_{1}}, \frac{\partial H_{0}}{\partial x_{2}}\right)^{T}$ and $\mathbf{u}(J)=\left(\phi_{m}(J), 0\right)^{T}$, then the transformation from $(\theta, J)$ to $\mathbf{x}$ is given by

$$
\mathbf{x}=\mathbf{g}\left(\frac{\theta}{\omega_{s}(J)}, \mathbf{u}(J)\right)
$$

The equations of motion can now be derived from the new Hamiltonian:

$$
\mathcal{H}(\theta, J, t)=h(J)+\epsilon H_{1}\left(\mathbf{g}\left(\frac{\theta}{\omega_{s}(J)}, \mathbf{u}(J)\right), t\right),
$$

where $\epsilon$ can be viewed as the size of the noise. This approach avoids the multivaluedness problem that occurs in the usual approach using mixed generating functions. This transformation procedure works more generally for a symmetric bowl potential $U$; details will be presented in Reference 6 .

If we write the $J$ equation as

$$
\dot{J}=-\frac{\partial \mathcal{H}}{\partial \theta}=: \epsilon\left(a(t) R_{1}(J, \theta)+\dot{\psi}(t) R_{2}(J, \theta)\right),
$$


then integrating and expanding in $\epsilon$ formally gives

$$
J_{T}-J_{0}=\epsilon \int_{0}^{T}\left[a(t) R_{1}\left(J_{0}, \theta_{0}+\omega_{s}\left(J_{0}\right) t\right)+\dot{\psi}(t) R_{2}\left(J_{0}, \theta_{0}+\omega_{s}\left(J_{0}\right) t\right)\right] d t+O\left(\epsilon^{2}\right),
$$

where $J_{T}:=J(T)$ and we have used the fact that $J(t)=J_{0}+O(\epsilon)$ and $\theta(t)=$ $\theta_{0}+\omega_{s}\left(J_{0}\right) t+O(\epsilon)$, for $T=O(1)$. We now assume that particles are uniformly distributed in $\theta_{0}$ so that $\theta_{0}$ can be viewed as a uniformly distributed random variable on $(0,2 \pi)$. This is equivalent to a statistical equilibrium assumption of uniformity on thin energy shells and should be the case after several synchrotron periods because the nonlinearity gives rise to phase randomization (filamentation) ${ }^{7}$ Also we consider the case where $\theta_{0}, a(t)$, and $\dot{\psi}(t)$ are independent. Squaring and taking the expected value, we obtain

$$
\begin{aligned}
& \frac{E\left[\left(J_{T}-J_{0}\right)^{2}\right]}{T} \\
=\epsilon^{2} & \frac{1}{T} \int_{0}^{T} \int_{0}^{T}\left\{C_{a}(t-s)\left\langle R_{1}\left(J_{0}, \theta_{0}\right) R_{1}\left(J_{0}, \theta_{0}+\omega_{s}\left(J_{0}\right)(s-t)\right)\right\rangle\right. \\
& \left.+C_{\dot{\psi}}(t-s)\left\langle R_{2}\left(J_{0}, \theta_{0}\right) R_{2}\left(J_{0}, \theta_{0}+\omega_{s}\left(J_{0}\right)(s-t)\right)\right\rangle\right\} d t d s+O\left(\epsilon^{3}\right) \\
=: & \epsilon^{2} \frac{1}{T} \int_{0}^{T}\left(\int_{0}^{T} w(t-s) d t\right) d s+O\left(\epsilon^{3}\right),
\end{aligned}
$$

where $E[]$ denotes the ensemble average, \langle\rangle denotes the average over $\theta_{0}$, and $C_{f}(\tau)=E[f(t) f(t+\tau)]$ is the auto-covariance of a stationary random process $f(t)$. Note that $C_{f}(-\tau)=C_{f}(\tau)$. In what follows we assume $C_{f}$ has whatever properties are necessary for our calculations to make sense. Integrating by parts gives

$$
\frac{1}{T} \int_{0}^{T}\left(\int_{0}^{T} w(t-s) d t\right) d s=\int_{-T}^{T} w(t) d t-\frac{1}{T} \int_{0}^{T} t w(t) d t+\frac{1}{T} \int_{-T}^{0} t w(t) d t
$$

Let $t_{c}$ be a measure of the correlation time; then for $T \gg t_{c}$, the last two terms on the right-hand side of Equation (2.17) are very small and the first term is $\int_{-\infty}^{\infty} w(t) d t$. Thus

$$
\begin{aligned}
\frac{E\left[\left(J_{T}-J_{0}\right)^{2}\right]}{T} \simeq & \epsilon^{2} \int_{-\infty}^{\infty}\left\{C_{a}(t)\left\langle R_{1}\left(J_{0}, \theta_{0}\right) R_{1}\left(J_{0}, \theta_{0}+\omega_{s}\left(J_{0}\right) t\right)\right\rangle\right. \\
& \left.+C_{\dot{\psi}}(t)\left\langle R_{2}\left(J_{0}, \theta_{0}\right) R_{2}\left(J_{0}, \theta_{0}+\omega_{s}\left(J_{0}\right) t\right)\right\rangle\right\} d t \\
= & : A\left(J_{0}\right) .
\end{aligned}
$$


Note that the function $\mathrm{A}$ is independent of $T$ and $\theta_{0}$ and thus depends only on $J_{0}$, and we have dropped the $O\left(\epsilon^{3}\right)$ term. The derivation here is basically that in Dôme. ${ }^{4} \mathrm{~A}$ lengthier calculation in the same reference shows

$$
\frac{E\left[J_{T}-J_{0}\right]}{T} \simeq \frac{1}{2} A^{\prime}\left(J_{0}\right)
$$

If we subdivide the time axis in intervals $\Delta t$ such that $t_{c} \ll T \ll \Delta t<1 / \epsilon^{2}$, then $J(t)$ does not vary much during $\Delta t$, a time interval during which $a(t)$ and $\psi(t)$ have forgotten their past. Thus in the time scale defined by $\Delta t$, the process $J(t)$ is expected to be approximately Markovian with infinitesimal mean and variance given by Equations (2.19) and (2.18), respectively. The latter follows because $T \gg t_{c}$ allows the steps from Equation (2.16) to Equation (2.18), and $T \ll \Delta t$ allows Equations (2.18) and (2.19) to be viewed as infinitesimal moments. The Fokker-Planck equation for the Markov process is thus

$$
\frac{\partial \rho}{\partial t}=-\frac{\partial}{\partial J}\left[\frac{1}{2} A^{\prime}(J) \rho\right]+\frac{1}{2} \frac{\partial^{2}}{\partial J^{2}}[A(J) \rho] .
$$

This reduces to Equation (2.4) with $D(J)=\frac{1}{2} A(J)$. Putting this on a rigorous mathematical basis is the goal of Reference 6 (see also the last paragraph of this section).

In the case where the correlation time is short relative to the synchrotron period, $t_{c} \ll 2 \pi / \omega_{s}(J)$, Equation (2.18) becomes

$$
\begin{aligned}
& D^{\text {white }}(J) \\
& =\frac{1}{2} S_{a}(0)\left\langle R_{1}\left(J, \theta_{0}\right)^{2}\right\rangle-\frac{1}{2} \omega_{s}^{2}(J) S_{\psi}(0)\left\langle R_{2}\left(J, \theta_{0}\right) \frac{\partial^{2} R_{2}}{\partial \theta_{0}^{2}}\left(J, \theta_{0}\right)\right\rangle \\
& =\frac{\sqrt{2}}{\pi \omega_{s}(J)} \int_{0}^{\phi_{m}(J)}\left[S_{a}(0) U^{\prime}(\phi)^{2}+S_{\psi}(0) U^{\prime \prime}(\phi)^{2}\right] \sqrt{U\left(\phi_{m}(J)\right)-U(\phi)} d \phi \\
& =: D_{a}^{\text {white }}+D_{\psi}^{\text {white }} .
\end{aligned}
$$

Here $S_{f}(\omega)$ and $C_{f}(t)$ are Fourier transform pairs with $S_{f}(\omega)=$ $\int_{-\infty}^{\infty} e^{i \omega t} C_{f}(t) d t=2 \int_{0}^{\infty} \cos (\omega t) C_{f}(t) d t$ and $C_{f}(t)=\int_{-\infty}^{\infty} e^{-i \omega t} S_{f}(\omega) \frac{d \omega}{2 \pi}=$ $\frac{1}{\pi} \int_{0}^{\infty} \cos (\omega t) S_{f}(\omega) d \omega$. Note that $E\left[f^{2}(t)\right]=C_{f}(0)=\frac{1}{\pi} \int_{0}^{\infty} S_{f}(\omega) d \omega$. In going from (2.18) to (2.21) we use the facts that $C_{\dot{\psi}}(t)=-C_{\psi}^{\prime \prime}(t)$, which follows from

$$
\begin{array}{r}
E\left[\frac{\psi(t+h)-\psi(t)}{h} \frac{\psi(t+\tau+h)-\psi(t+\tau)}{h}\right] \\
=-\frac{C_{\psi}(\tau+h)-2 C_{\psi}(\tau)+C_{\psi}(\tau-h)}{h^{2}}
\end{array}
$$


in the limit as $h \rightarrow 0$, and that $\int_{-\infty}^{\infty} C_{f}(t) g(t) d t \simeq g(0) \int_{-\infty}^{\infty} C_{f}(t) d t=g(0) S_{f}(0)$ for $t_{c} \ll 2 \pi / \omega_{s}(J)$. Because in the latter case $C_{f}$ behaves like a delta function we will refer to this as the white-noise case.

Let $R_{l}(J, \theta)=\sum_{m=-\infty}^{\infty} R_{l, m}(J) e^{i m \theta}, l=1,2$; then it is easy to show that

$$
D(J)=\sum_{m>0}\left\{\left|R_{1, m}(J)\right|^{2} S_{a}\left(m \omega_{s}(J)\right)+\left|R_{2, m}(J)\right|^{2} S_{\dot{\psi}}\left(m \omega_{s}(J)\right)\right\},
$$

where we have used the fact that $R_{l, 0}=0$. In the pendulum case (sinusoidal RF voltage),

$$
\begin{aligned}
D(J) & =4 \sum_{m=2,4, \ldots}^{\infty} \frac{\left(m \omega_{s}\right)^{4}}{\sinh ^{2}(m v)} S_{a}\left(m \omega_{s}\right)+4 \sum_{m=1,3, \ldots}^{\infty} \frac{\left(m \omega_{s}\right)^{4}}{\cosh ^{2}(m v)} S_{\psi}\left(m \omega_{s}\right) \\
& =: D_{a}(J)+D_{\psi}(J) .
\end{aligned}
$$

The quantities $J, \omega_{s}(J)$, and $v(J)$ are easily defined through the intermediate variable $k, 0 \leq k<1$, by $J=\left(8 \Omega k^{2} / \pi\right) B(k), \omega_{s}=\Omega(\pi / 2 K(k))$, and $v=(\pi / 2) K\left(\sqrt{1-k^{2}}\right) / K(k)$, where $K$ is the complete elliptic integral of the first kind and $B(k)=\int_{0}^{\pi / 2} \cos ^{2} x d x / \sqrt{1-k^{2} \sin ^{2} x}$. The variable $k$ is related to the unperturbed energy $h$ by $h=2 k^{2} \Omega^{2}$. See Reference 4 for further details.

In Figure 1(a) we present $D_{a}(J)$ for white amplitude noise; that is, $S_{a}(\omega)=$ constant. It is easy to show that $D_{a}(J) \propto J^{2}$ for small $J$. Since the equation of motion for small $J$ is $\ddot{\phi}+\Omega^{2}(1+a(t)) \phi=0$, one might, from the theory of the Mathieu equation, expect resonant-type behavior due to Fourier components of $a(t)$ near $2 \Omega$. In Figure 1(b), we present $D_{a}(J)$ for notched amplitude noise; that is, $S_{a}(\omega)$ is a non-zero constant except in a region about $\omega=2 \Omega$ where it is zero. In this case, $D_{a}(J) \propto J^{4}$ for small $J$, showing that $D_{a}(J)$ at small $J$ does indeed depend quite sensitively on $S_{a}(\omega)$ near $\omega=2 \Omega$.

In Figure 2(a), we present $D_{\psi}(J)$ for white phase noise; that is, $S_{\psi}(\omega)=$ constant. Again, it is easy to show that $D_{\psi}(J) \propto J$ for small $J$. Since the equation of motion for small $J$ is $\ddot{\phi}+\Omega^{2} \phi=\ddot{\psi}(t)$, one might expect a decrease in $D_{\psi}(J)$ for small $J$ if the Fourier components of $\psi(t)$ near $\omega=\Omega$ are filtered out. This is indeed the case, as shown in Figure 2(b), where we present $D_{\psi}(J)$ for notched phase noise; that is, $S_{\psi}(\omega)$ is a non-zero constant except in a region about $\omega=\Omega$ where it is zero. In this case, it can be shown that $D_{\psi}(J)$ behaves like $J^{3}$ for small $J$.

The frequency, $\omega_{s}(J)$, approaches 0 as $J$ approaches $J_{s}$, so the assumptions used in deriving the theory are probably not valid near the separatrix. This is reflected in the white-noise case in the fact that $D(J)$ approaches $\infty$ like $1 / \omega_{s}(J)$ as $J \nearrow J_{s}$. Our numerical results indicate that $D(J)$ approaches $\infty$ more generally, but we have not found conditions on $S$ in (2.23) that guarantee this. To pursue this, we looked at the process in terms of energy. Since $J(t)$ is approximately Markovian, so is the energy, $e(t)$ :

$$
e(t)=h(J(t))
$$




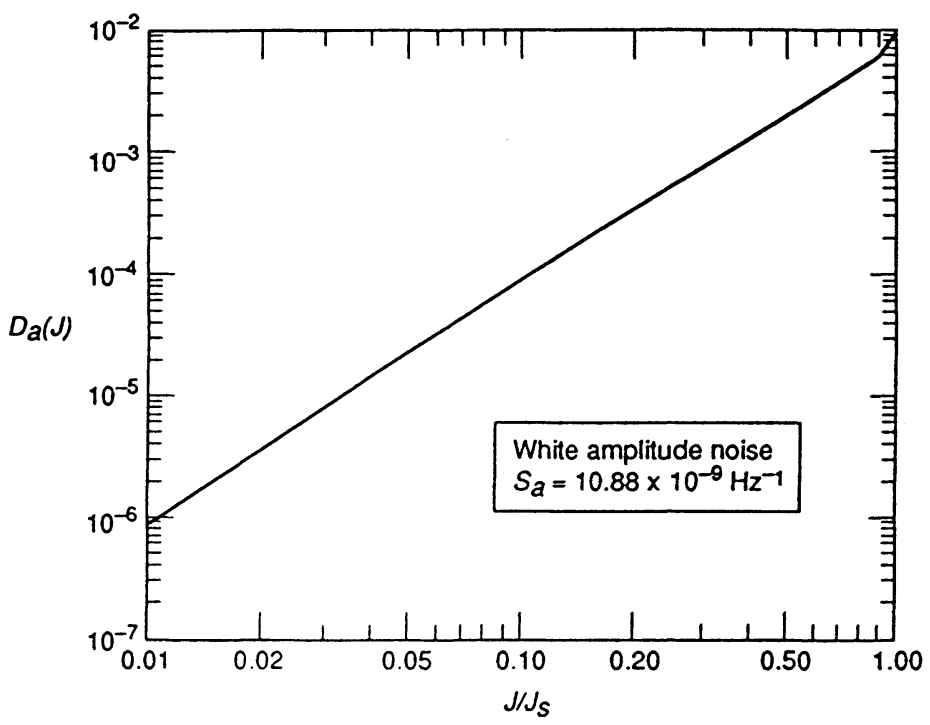

FIGURE 1a:

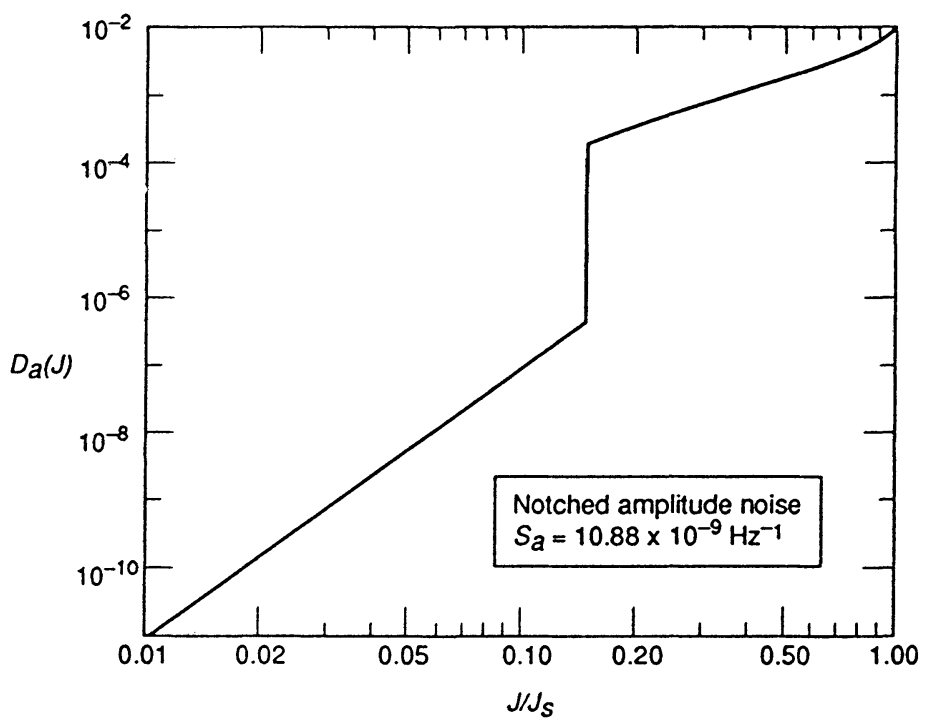

FIGURE 1b:

FIGURE 1: Diffusion Coefficient for Amplitude Noise With $S_{a}=10.88 \times 10^{-9} \mathrm{~Hz}^{-1}$. (a) White noise. (b) Notched noise. 


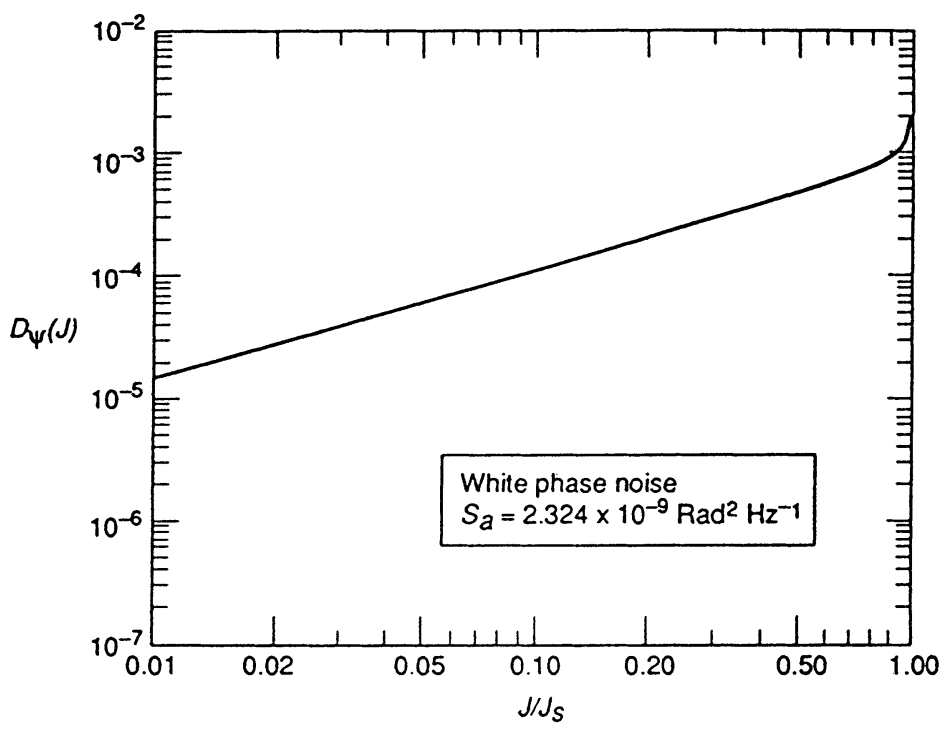

FIGURE 2a:

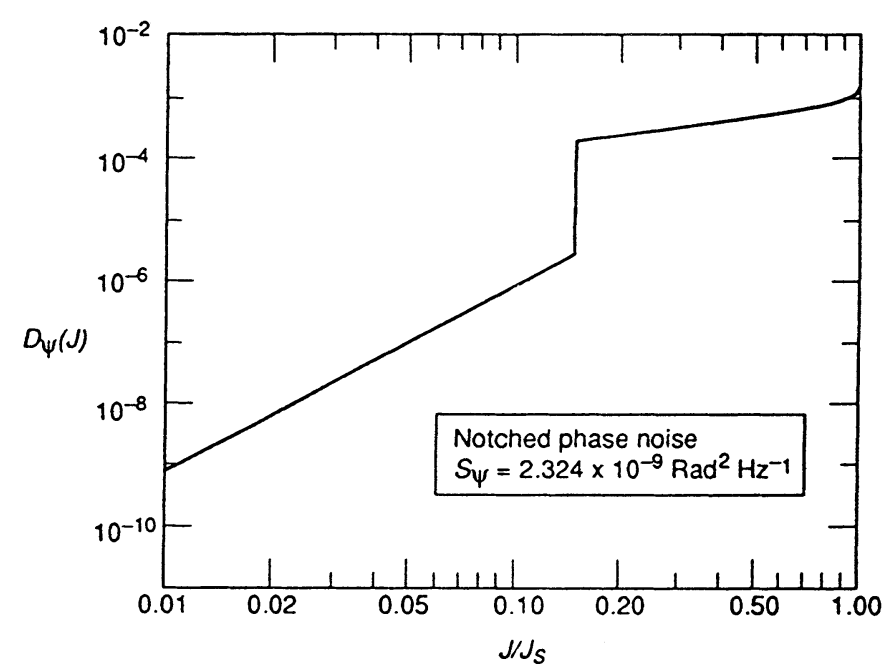

FIGURE 2b:

FIGURE 2: Diffusion Coefficient for Phase Noise with $S_{\psi}=2.324 \times 10^{-9} \mathrm{rad}^{2} \mathrm{~Hz}^{-1}$. (a) White noise. (b) Notched noise. 
The transformation $e=h(J)$ and $\rho_{e}(e)=\rho(J) \omega_{s}(J)$ gives the Fokker-Planck equation in energy,

$$
\frac{\partial \rho_{e}}{\partial t}=-\frac{\partial}{\partial e}\left[A_{1}(e) \rho_{e}\right]+\frac{1}{2} \frac{\partial^{2}}{\partial e^{2}}\left[A_{2}(e) \rho_{e}\right],
$$

where $A_{1}(e)=\left.\left(D(J) \omega_{s}(J)\right)^{\prime}\right|_{J=J(e)}$ and $A_{2}(e)=2 D(J(e)) \omega_{s}^{2}(J(e))$. There are two other ways to see this. One is to note that Itô's formula transforms the Itô equation, ${ }^{8}$

$$
d J=D^{\prime}(J) d t+\sqrt{2 D(J)} d W
$$

into

$$
d e=A_{1}(e) d t+\sqrt{A_{2}(e)} d W,
$$

where $W(t)$ is standard Brownian motion. The other way is to note that

$$
h\left(J_{T}\right)-h\left(J_{0}\right) \cong h^{\prime}\left(J_{0}\right)\left(J_{T}-J_{0}\right)+\frac{1}{2} h^{\prime \prime}\left(J_{0}\right)\left(J_{T}-J_{0}\right)^{2}
$$

gives the corresponding expressions for $E\left[\frac{\left(e_{T}-e_{0}\right)^{2}}{T}\right]$ and $E\left[\frac{e_{T}-e_{0}}{T}\right]$. Thus in the white-noise case the diffusion coefficient $A_{2}(e)$ approaches zero as $J \nearrow J_{s}$, further illustrating problems at the separatrix. At any rate, we are investigating the possibility of a diffusion theory valid across the separatrix.

The DKW diffusion theory is heuristic in the sense that the stochastic process $J(t)$ is assumed to be approximately Markovian on a time scale large compared to the correlation times of $a(t)$ and of $\psi(t)$. Under this assumption and the assumption of uniformity in $\theta$, the derivation of the diffusion coefficient seems reasonable. The Markov approximation will be discussed in detail in Reference 6, where the probabilistic limit theorems developed in Reference 9 will be applied to the rf noise problem. These theorems give conditions under which the process $J(t)$ is approximately Markovian. Here approximation is in the sense of weak convergence, an important idea from modern probability. In Section 3.0 we show that the DKW diffusion theory is reasonable at the SSC energy by comparing it with a simulation study based on Equation (2.1).

\section{NUMERICAL SOLUTIONS AND COMPARISON WITH TRACKING SIMU- LATIONS}

It is straightforward to integrate the diffusion equation (2.4) by the method of lines. That is, the action variable $J$ is discretized, the partial derivatives with respect to $J$ are calculated by a finite-difference approximation, and an ODE integrator with automatic error control is employed to obtain the evolution in time of the action density at each grid point of $J$. Before integrating Equation (2.4) one must calculate the diffusion coefficient $D(J)$ and the initial action density $\rho_{0}(J)$. 
To calculate the diffusion coefficient $D(J)$, we use Equation (2.21) in the white noise case or Equation (2.23) for a particular noise spectral density. We assume the following spectral densities for low pass and notched phase noise: (1) $S_{\psi}^{\text {low pass }}=$ constant for $0<\omega<r \Omega$ and 0 elsewhere, (2) $S_{\psi}^{\text {notched }}=0$ for $r \Omega<\omega<\Omega$ and constant elsewhere; and we assume the following for low-pass and notched amplitude noise: (1) $S_{a}^{\text {low pass }}=$ constant for $0<\omega<2 r \Omega$ and 0 elsewhere, (2) $S_{a}^{\text {notched }}=0$ for $2 r \Omega<\omega<2 \Omega$ and constant elsewhere. Here $r$ is the filtering fraction with $0<r<1$. For white phase and amplitude noise, the following expressions for $D$ can be derived from Equation (2.21) (they also follow from (2.23)):

$$
\begin{aligned}
D_{\psi}^{\text {white }} & =\frac{8}{\pi^{2}} \Omega^{4} S_{\psi} k^{2} K(k)\left(B(k)-4 k^{2} B_{1}(k)\right), \\
D_{a}^{\text {white }} & =\frac{32}{\pi^{2}} \Omega^{4} S_{a} k^{4} K(k) B_{1}(k),
\end{aligned}
$$

where $B_{1}(k)=\int_{0}^{\pi / 2} \sin ^{2} x \cos ^{2} x \sqrt{1-k^{2} \sin ^{2} x} d x$. For white noise, we assume the spectral density extends to $f_{0} / 2$ in frequency (this will later be justified by Equation (3.11)). Thus, in Equation (3.1) $S_{f}=T_{0} \sigma_{f}^{2}$, where $T_{0}=\frac{1}{f_{0}}$ and $\sigma_{f}^{2}=\operatorname{var}\{f\}$. For low-pass phase and amplitude noise, $D$ is computed according to

$$
\begin{aligned}
& D_{\psi}^{\text {low pass }}=4 \omega_{s}^{4} S_{\psi} \sum_{m=1}^{n_{1}} \frac{(2 m-1)^{4}}{\cosh ^{2}((2 m-1) v)}, \\
& D_{a}^{\text {low pass }}=4 \omega_{s}^{4} S_{a} \sum_{m=1}^{n_{1}-1} \frac{(2 m)^{4}}{\sinh ^{2}(2 m v)},
\end{aligned}
$$

where $n_{1}$ is an integer such that $\left(2 n_{1}-1\right) \omega_{s} \leq r \Omega<\left(2 n_{1}+1\right) \omega_{s}$ for phase noise or $2\left(n_{1}-1\right) \omega_{s} \leq 2 r \Omega<2 n_{1} \omega_{s}$ for amplitude noise. In Equation (3.2) $S_{\psi}=\frac{1}{f_{0}} \frac{\sigma_{\psi}^{2}}{2 r Q_{s o}}$ and $S_{a}=\frac{1}{f_{0}} \frac{\sigma_{a}^{2}}{4 r Q_{s o}}$. Here $Q_{s o}$ is the small-oscillation synchrotron tune. For the SSC, $Q_{s o}=0.00123$. For notched phase and amplitude noise, $D$ is computed according to

$$
\begin{aligned}
D_{\psi}^{\text {notched }} & =D_{\psi}^{\text {white }}-4 \omega_{s}^{4} S_{\psi} \sum_{m=n_{1}+1}^{n_{2}} \frac{(2 m-1)^{4}}{\cosh ^{2}((2 m-1) v)}, \\
D_{a}^{\text {notched }} & =D_{a}^{\text {white }}-4 \omega_{s}^{4} S_{a} \sum_{m=n_{1}}^{n_{2}-1} \frac{(2 m)^{4}}{\sinh ^{2}(2 m v)}
\end{aligned}
$$

where $n_{2}$ is an integer such that $\left(2 n_{2}-1\right) \omega_{s} \leq \Omega<\left(2 n_{2}+1\right) \omega_{s}$ for phase noise or $2\left(n_{2}-1\right) \omega_{s} \leq 2 \Omega<2 n_{2} \omega_{s}$ for amplitude noise. In Equation (3.3) $S_{\psi}=\frac{1}{f_{0}} \frac{\sigma_{\psi}^{2}}{1-2(1-r) Q_{s o}}$ 
and $S_{a}=\frac{1}{f_{0}} \frac{\sigma_{a}^{2}}{1-4(1-r) Q_{s o}}$. Since we obtain the diffusion coefficient and the action as functions of $k$, we spline-fit $D(k)$ versus $J(k)$ in order to obtain $D$ as a function of $J$.

In calculating the initial action density we have taken a Gaussian distribution in $P$ and $\phi$ matched to the small-amplitude synchrotron ellipses. Since the longitudinal emittance at the SSC is small, the initial action density is, to a good approximation,

$$
\rho_{0}(J)=\frac{1}{\mu} e^{-J / \mu}, \quad \text { with } \quad \mu=\Omega\left(\frac{2 \pi h \sigma_{l}}{C_{0}}\right)^{2},
$$

where $\sigma_{l}$ is the rms longitudinal bunch spread. More generally we can start with an arbitrary initial density $p(P, \phi)$. (The nonlinearity of the pendulum will cause a uniformization on thin energy shells. $)^{7}$ The calculation for the resultant action density follows, where for convenience we assume symmetry in both $P$ and $\phi$. Let $\rho_{H}(h)$ be the density in energy; then

$$
\int_{0}^{h} \rho_{H}(x) d x=4 \int_{0}^{\phi_{m}} d \phi \int_{0}^{\sqrt{2(h-U(\phi))}} p(P, \phi) d P .
$$

Differentiating, we obtain

$$
\rho_{H}(h)=4 \int_{0}^{\phi_{m}} \frac{p\left(P_{h}(\phi), \phi\right)}{P_{h}(\phi)} d \phi,
$$

where $P_{h}(\phi)=\sqrt{2(h-U(\phi))}$. The action density $\rho_{0}(J)$ is related to $\rho_{H}(h)$ by $\rho(J) d J=\rho_{H}(h) d h$; thus

$$
\rho_{0}(J)=\rho_{H}(h) \frac{d h}{d J}=\rho_{H}(h) \omega_{s}(J) .
$$

We have used this procedure in our calculations.

In the white-amplitude-noise case the action density at $J=0$ is fixed and the slope there steepens exponentially with time (from Equation (2.4) at $J=0, \frac{\partial \rho}{\partial t}=0$ and $\left.\frac{\partial}{\partial t} \frac{\partial \rho}{\partial J}=D^{\prime \prime}(0) \frac{\partial \rho}{\partial J}\right)$. Because of this, we have used a variable grid, with finer mesh near $J=0$, for discretizing the partial derivatives with respect to $J$. In the notched-noise cases where the filtered spectral density results in a discontinuous diffusion coefficient, we have imposed an additional finite-difference equation at the discontinuity point to preserve conservation of probability at that point. We have verified our numerical results by (1) using a Galerkin (finite element) approach with piecewise linear basis functions, and (2) comparing with exact solutions that are known in the $D(J)=J$ and $J^{2}$ cases. Our numerical study is detailed in Reference 10.

We have conducted the tracking simulations using a linear lattice for the SSC. In order to apply the results to the extraction problem (to be discussed in Section 4.2), tracking is done in the full 6-dimensional phase space: $x, x^{\prime}, y, y^{\prime}, l, \delta$. Here $x$ is the horizontal coordinate, $x^{\prime}$ the horizontal angle, $y$ the vertical coordinate, $y^{\prime}$ the vertical angle, $l$ the deviation in path length, and $\delta=\Delta p / p_{s}$, the fractional deviation in momentum. The initial values of each variable are randomly generated according to Gaussian distributions with the rms values of $x, x^{\prime}, y$, and $y^{\prime}$ determined by the lattice 
functions, and the rms values of $l$ and $\delta$ determined by assuming an energy spread of $1 \mathrm{GeV}$. To carry the protons around the ring, the following transfer map from path length $s_{0}$ to $s$ is constructed using the lattice functions:

$$
\left(\begin{array}{cccccc}
C_{x} & S_{x} & 0 & 0 & 0 & E \\
C_{x}^{\prime} & S_{x}^{\prime} & 0 & 0 & 0 & E^{\prime} \\
0 & 0 & C_{y} & S_{y} & 0 & 0 \\
0 & 0 & C_{y}^{\prime} & S_{y}^{\prime} & 0 & 0 \\
F & G & 0 & 0 & 1 & \alpha_{c} \Delta s-\left(F \eta_{0}+G \eta_{0}^{\prime}\right) \\
0 & 0 & 0 & 0 & 0 & 1
\end{array}\right)
$$

with

$$
\begin{aligned}
C & =\sqrt{\frac{\beta}{\beta_{0}}}\left(\cos 2 \pi Q+\alpha_{0} \sin 2 \pi Q\right), \\
S & =\sqrt{\beta \beta_{0}} \sin 2 \pi Q, \\
C^{\prime} & =\frac{1}{\sqrt{\beta \beta_{0}}}\left(\left(\alpha_{0}-\alpha\right) \cos 2 \pi Q-\left(1+\alpha \alpha_{0}\right) \sin 2 \pi Q\right), \\
S^{\prime} & =\sqrt{\frac{\beta_{0}}{\beta}}(\cos 2 \pi Q-\alpha \sin 2 \pi Q), \\
E & =\eta-C_{x} \eta_{0}-S_{x} \eta_{0}^{\prime}, \\
E^{\prime} & =\eta^{\prime}-C_{x}^{\prime} \eta_{0}-S_{x}^{\prime} \eta_{0}^{\prime}, \\
F & =C_{x} \eta^{\prime}-C_{x}^{\prime} \eta-\eta_{0}^{\prime}, \\
G & =S_{x} \eta^{\prime}-S_{x}^{\prime} \eta+\eta_{0},
\end{aligned}
$$

where $\Delta s=s-s_{0}, Q$ is the tune advance from $s_{0}$ to $s, \alpha$ and $\beta$ are the usual CourantSnyder parameters evaluated at $s, \eta$ is the dispersion function evaluated at $s$, and the subscript 0 denotes the lattice functions evaluated at $s_{0}$. In general, there is a coupling in the map (3.7) between horizontal and longitudinal motions. For comparisons with the diffusion-in-action theory, a one-turn map with $s=s_{0}+C_{0}$ is sufficient. If the dispersion is negligible at the rf cavity, the coupling between horizontal and longitudinal motions in the one-turn map evaluated at the rf cavity vanishes. In this case, we obtain $l_{n+1}=l_{n}+\alpha_{c} C_{0} \delta_{n}$ and thus Equation (2.1a) by using the relationship

$$
\phi_{n}=(2 \pi h) l_{n} / C_{0}+\psi_{n} .
$$

For bent-crystal extraction, the crystal and rf cavity are at different locations, and two maps are needed to carry the bunch once around the ring. Table 2 lists the relevant parameters for constructing transfer maps in the tracking simulations. Table 3 lists the standard deviations in $x, x^{\prime}, y, y^{\prime}, l$, and $\delta$ of the SSC beam. 
TABLE 2: Relevant Parameters for Constructing Transfer Maps.

\begin{tabular}{lll}
\hline parameter & @bent crystal & @rf cavity \\
\hline$\alpha_{x}$ & 1.773 & -2.996 \\
$\alpha_{y}$ & 0.734 & -0.112 \\
$\beta_{x}$ & $1385.09 \mathrm{~m}$ & $440.25 \mathrm{~m}$ \\
$\beta_{y}$ & $544.79 \mathrm{~m}$ & $44.62 \mathrm{~m}$ \\
$\eta$ & $-4 . \mathrm{m}$ & $0 . \mathrm{m}$ \\
$\eta^{\prime}$ & 0. & 0. \\
$Q_{x}$ & $61.269^{a}$ & $61.515^{b}$ \\
$Q_{y}$ & $60.994^{a}$ & $61.783^{b}$ \\
$\Delta s$ & $43338.58^{a} \mathrm{~m}$ & $43781.42^{b} \mathrm{~m}$ \\
\hline
\end{tabular}

${ }^{a}$ from bent crystal to rf cavity

${ }^{b}$ from bent crystal to rf cavity

TABLE 3: Standard Deviations in $x, x^{\prime}, y, y^{\prime}, l$, and $\delta$ of the SSC Beam.

\begin{tabular}{lll}
\hline r.m.s. & @bent crystal & @rf cavity \\
\hline$\sigma_{x}$ & $2.549 \times 10^{-4} \mathrm{~m}$ & $1.437 \times 10^{-4} \mathrm{~m}$ \\
$\sigma_{x^{\prime}}$ & $1.840 \times 10^{-7} \mathrm{rad}$ & $1.031 \times 10^{-6} \mathrm{rad}$ \\
$\sigma_{y}$ & $1.599 \times 10^{-4} \mathrm{~m}$ & $4.575 \times 10^{-5} \mathrm{~m}$ \\
$\sigma_{y^{\prime}}$ & $2.935 \times 10^{-7} \mathrm{rad}$ & $1.032 \times 10^{-6} \mathrm{rad}$ \\
$\sigma_{l}$ & $5.127 \times 10^{-2} \mathrm{~m}$ & $5.127 \times 10^{-2} \mathrm{~m}$ \\
$\sigma_{\delta}$ & $5 \times 10^{-5}$ & $5 \times 10^{-5}$ \\
\hline
\end{tabular}

When the protons traverse the rf cavity, the relative momentum difference is changed according to

$$
\delta_{n+1}=\delta_{n}-\frac{e V}{p_{s} v_{s}}\left(1+a_{n}\right) \sin \left(\frac{2 \pi h l_{n}}{C_{0}}+\psi_{n}\right) .
$$

Equation (3.10) is obtained by dividing Equation (2.1b) by $2 \pi h \eta$ and using Equation (3.9). In computer simulations, white phase or amplitude noise refers to the case where the $\psi_{n}$ or $a_{n}$ are drawn independently from a Gaussian distribution each time the bunch passes through the rf cavity. To compare this with the DKW theory, we need to define $f(t)$ from the $\left\{f_{n}\right\}$, where $f_{n}$ can be $\psi_{n}$ or $a_{n}$. If we let

$$
f(t)=\sum_{n=-\infty}^{\infty} \frac{\sin \frac{\pi}{T_{0}}\left(t-T_{0} n\right)}{\frac{\pi}{T_{0}}\left(t-T_{0} n\right)} f_{n},
$$

then $f\left(k T_{0}\right)=f_{k}$, and by the sampling theorem of Shannon, ${ }^{11} f(t)$ has zero spectral density outside $\left[-\pi / T_{0}, \pi / T_{0}\right]$ and a correlation time on the order of $T_{0}$. This is small 
compared to $2 \pi / \omega_{s}(J)$, and so the white-noise approximation of Equation (2.21) is valid with $S_{f}(0)=T_{0} \sigma_{f}^{2}$, where $\sigma_{f}^{2}=v a r\{f\}$. To obtain low-pass and notched phase noise, the following is done beforehand: generate a time series of white noise for $N$ turns, $\left\{\psi_{1}, \psi_{2}, \cdots, \psi_{N}\right\}$, compute the discrete Fourier series $\left\{\Phi_{1}, \Phi_{2}, \cdots, \Phi_{N}\right\}$, and construct

$$
\hat{\psi}_{n}=F_{1} \frac{2}{N} \sum_{m=2}^{m_{0}} \operatorname{Re}\left(\Phi_{m} e^{2 \pi i(m-1)(n-1) / N}\right)
$$

in the case of low-pass noise, or

$$
\hat{\psi}_{n}=F_{2}\left\{\psi_{n}-\frac{2}{N} \sum_{m=m_{0}+1}^{m_{1}} \operatorname{Re}\left(\Phi_{m} e^{2 \pi i(m-1)(n-1) / N}\right)\right\}
$$

in the case of notched noise. Here $m_{0} \geq 2$ is the largest integer such that $m_{0} \leq Q_{s o} N r$, $m_{1}$ is the smallest integer such that $m_{1} \geq Q_{s o} N$, and $F_{1}$ and $F_{2}$ are normalization factors such that $\psi_{n}$ and $\hat{\psi}_{n}$ have the same $\sigma_{\psi}$. With $n$ being the turn number, and the synchrotron tune for $\Phi_{m} \exp (2 \pi i(m-1)(n-1) / N)$ being $Q_{m}=(m-1) / N$, Equations (3.12) and (3.13) say that low-pass phase noise contains only the terms whose synchrotron tunes are less than $r Q_{s o}$, and notched phase noise contains all the terms except those whose tunes are between $r Q_{s o}$ and $Q_{s o}$. Low-pass and notched amplitude noise are also obtained from Equations (3.12) and (3.13) except that $m_{0}$ is the largest integer that satisfies $m_{0} \leq 2 Q_{s o} N r$, and $m_{1}$ is the smallest integer that satisfies $m_{1} \geq 2 Q_{s o} N$.

To compare the diffusion theory and the tracking simulation, we now present results for the white and notched cases. (The low-pass case will be discussed in the applications section.) The comparisons of action distribution and longitudinal emittance growth are shown in Figures 3(a) and 3(b), respectively, for white amplitude noise with $\sigma_{a}=0.2$, and in Figures 4(a) and 4(b) for notched amplitude noise with $\sigma_{a}=0.2$. Here the longitudinal emittance is defined as the average of the action over the surviving particles (recall that $2 \pi J$ is the area in phase space). The comparisons for white and notched phase noise with $\sigma_{\psi}=0.1$ are shown in Figures 5 and 6 , respectively. The agreement between simulation and theory is good in all cases. This gives us confidence in using the DKW diffusion theory in place of the more computationally intensive simulations.

\section{APPLICATIONS}

An important cause for loss of beam lifetime in the CERN Super Proton Sychrotron (SPS $)^{1,2}$ was shown to be the existence of phase noise associated with the radial loop, the beam phase measurement, and the low-level signal generators in the rf system. Noise in bands containing the small-amplitude synchrotron frequency was found to be especially deleterious. Because of the low synchrotron frequency in the SSC collider ring, this question was raised as a concern in the Conceptual Design Report ${ }^{12}$ (CDR), 


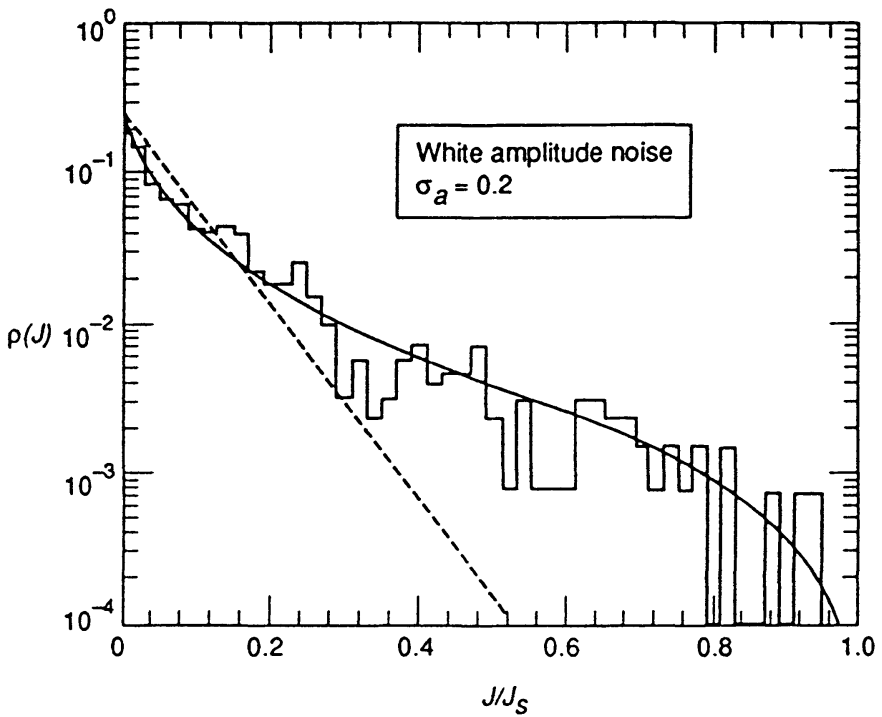

FIGURE 3a:

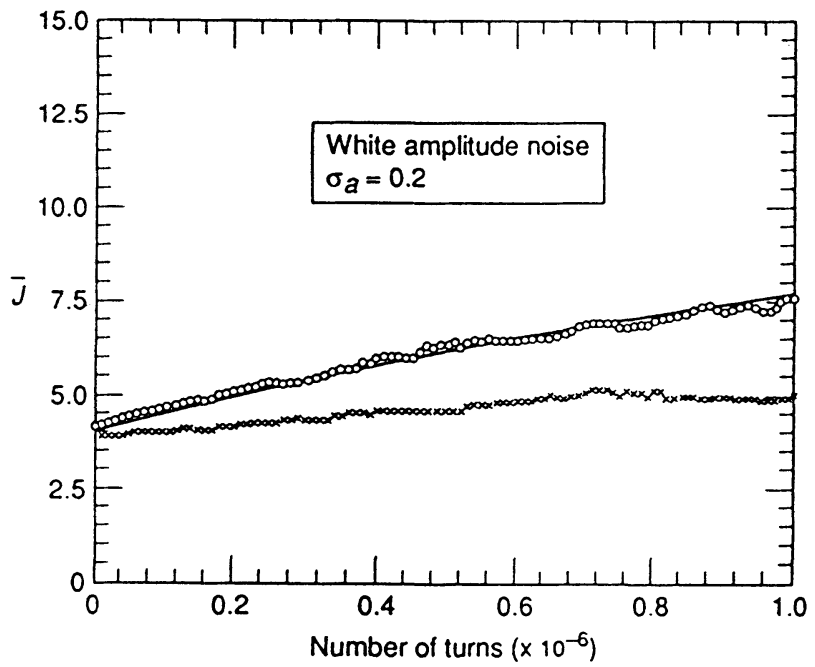

FIGURE 3b:

FIGURE 3: Comparison Between the DKW Diffusion Theory and the Tracking Simulation in the Case of White Amplitude Noise with $\sigma_{a}=0.2$. (a) Action density. Histogram: tracking simulation after $10^{6}$ turns. Solid curve: theory after $10^{6}$ turns. Dashed curve: initial density. (b) Longitudinal emittance vs. turn number. Curve: theory. Dots: tracking simulation without a bent crystal. A particle is regarded as lost when its $k$ value exceeds 0.96 , where $k$ is the variable defined by $h=2 k^{2} \Omega^{2}$. Crosses: tracking simulation with a bent crystal at $x=1 \mathrm{~mm}$. A particle is intercepted by the crystal and discarded when its $x$ value exceeds $1 \mathrm{~mm}$. 


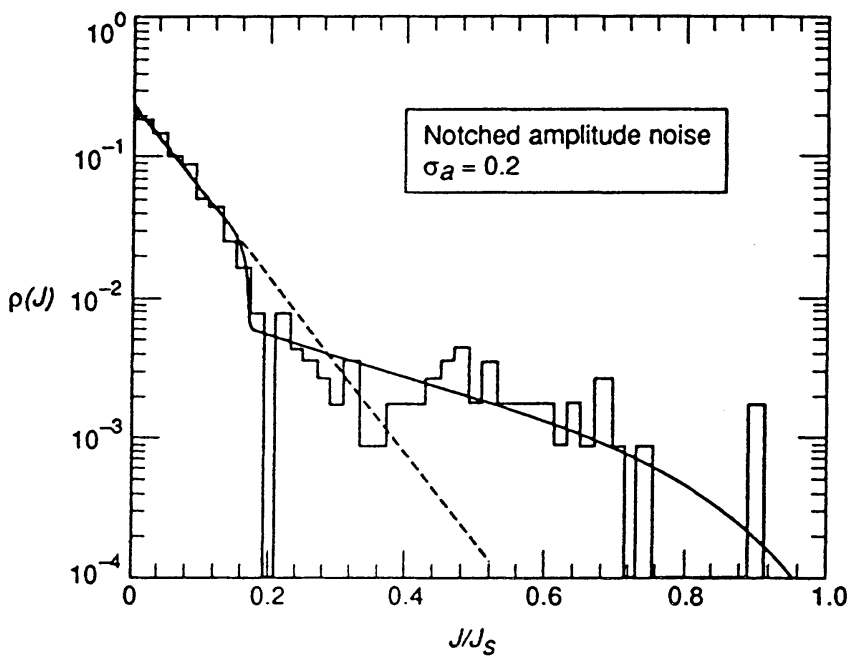

FIGURE 4a:

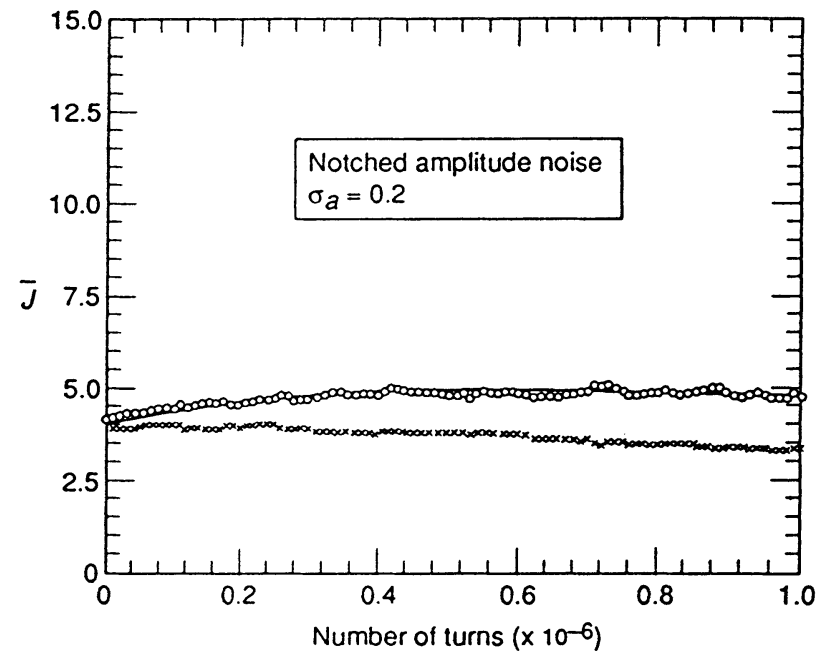

FIGURE 4b:

FIGURE 4: As in Figure 3 for Notched Amplitude Noise with $\sigma_{a}=0.2$. 


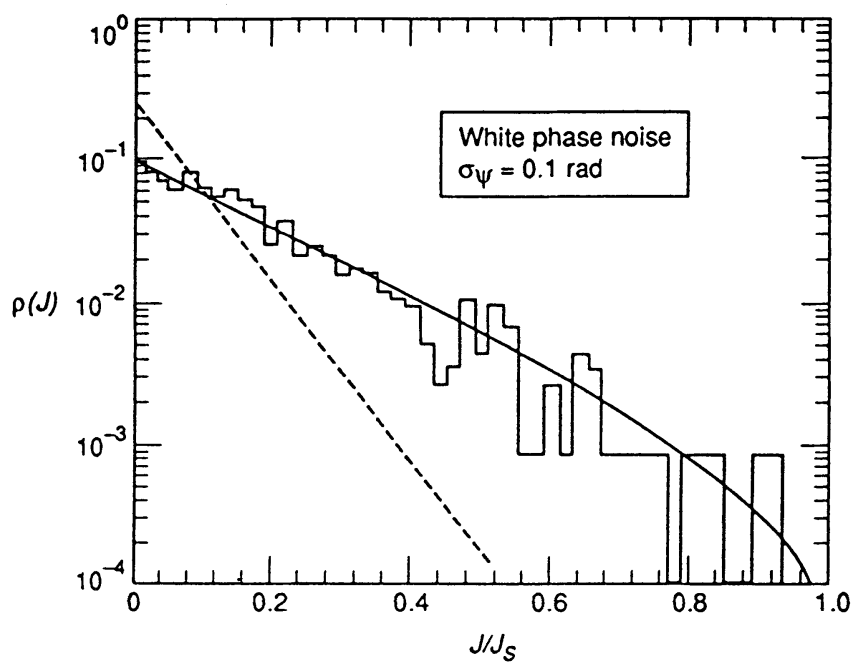

FIGURE 5a:

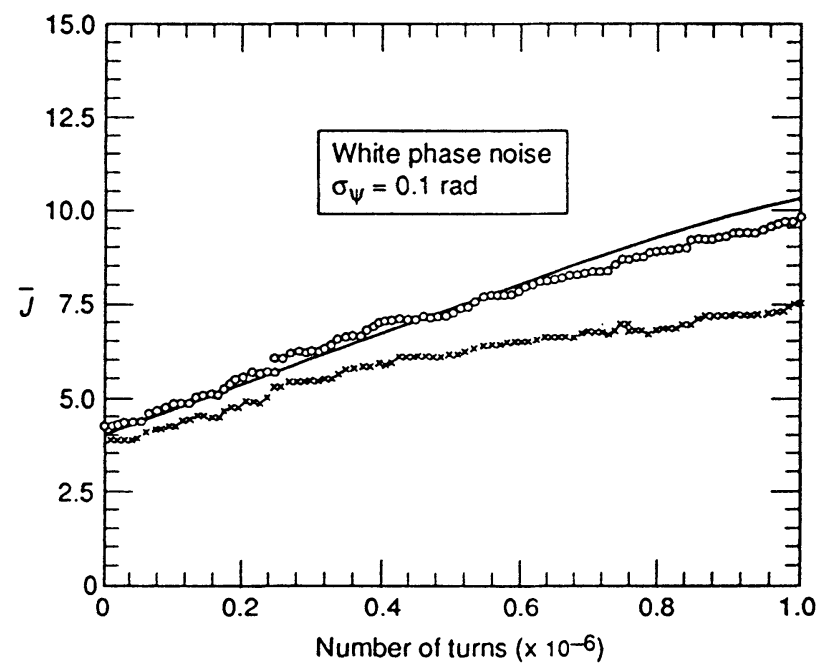

FIGURE 5b:

FIGURE 5: As in Figure 3 for White Phase Noise with $\sigma_{\psi}=0.1$. 


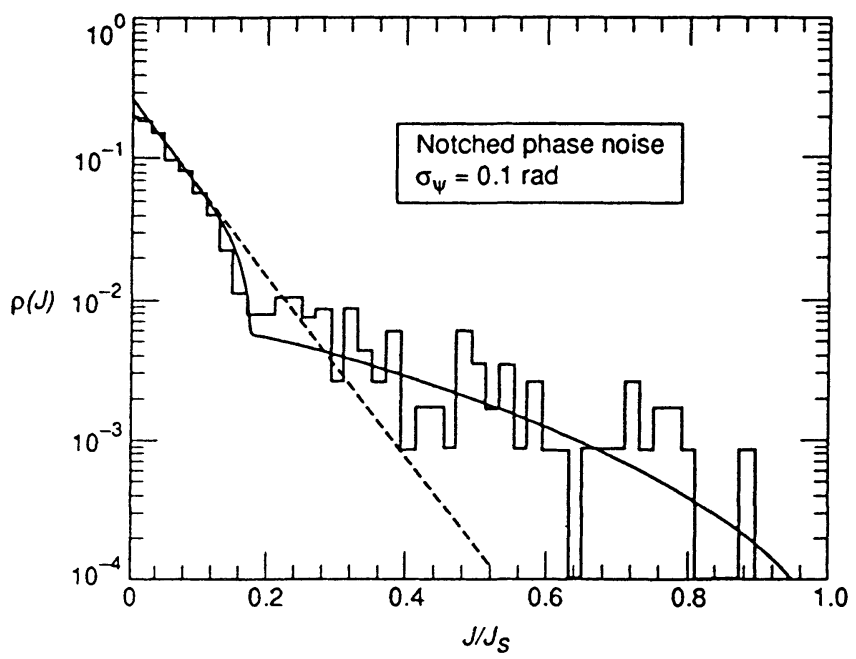

FIGURE 6a:

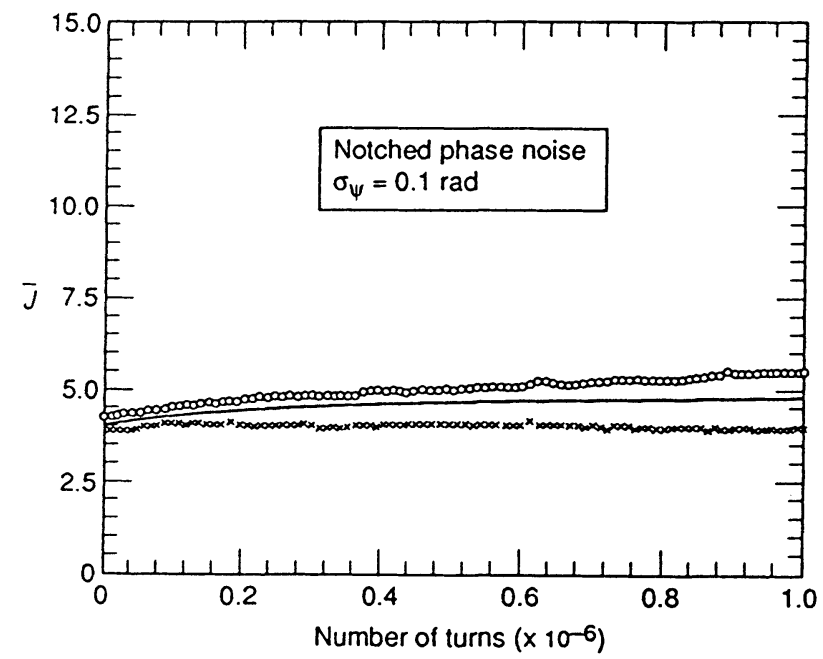

FIGURE 6b:

FIGURE 6: As in Figure 3 for Notched Phase Noise with $\sigma_{\psi}=0.1$. 
even for the very-high-quality signal synthesis systems available today. In Section 4.1, we discuss our results that address this issue.

The use of channeling in a bent single crystal, of Si for example, has been suggested $^{13,14}$ as one possibility for low-intensity beam extraction, and has recently been studied considerably ${ }^{15-18}$ for the SSC and for the Large Hadron Collider (LHC). A beam so extracted could feed a fixed-target experiment ${ }^{19}$ or provide, at the SSC, a $20-\mathrm{TeV}$ test beam. ${ }^{20}$ (There is no other provision for a test beam of this energy.) It is outside the scope of this paper to discuss the channeling physics by which an incident beam is deflected into the beamline by a bent crystal. Several good discussions are readily available. ${ }^{21}$ Here it is sufficient to note that deflection of a beam of highenergy particles in such a crystal has been demonstrated, ${ }^{22}$ as has extraction from an accelerator, ${ }^{23}$ albeit at low efficiency, and new experiments have been planned on the Tevatron at Fermilab ${ }^{24}$ and on the SPS. ${ }^{18}$ To extract continuously, some mechanism must be used to feed protons onto the crystal. At sufficiently high luminosity, the natural growth of halo should suffice; this is expected to be the case at the LHC. For the SSC, at $\mathcal{L}=10^{33} \mathrm{~cm}^{-2} \mathrm{sec}^{-1}$, the halo is not likely to be sufficient. In Section 4.2 we discuss our results on the application of noise injected into the rf system for this purpose.

\subsection{Noise in the RF System}

The low-level signal generator inevitably has a noise spectrum in the neighborhood of the carrier, which typically is falling and, for a high-quality generator, rapidly reaches an inconsequential level. Equation (2.23) shows that for phase noise only the spectral density at the odd harmonics of the synchrotron frequency contributes to the diffusion coefficient, whereas for amplitude noise only the even harmonics matter. In particular, for particles in the longitudinal core, only the spectral density evaluated at the first harmonic contributes significantly to the diffusion coefficient for phase noise, and only the second harmonic contributes significantly for amplitude noise. Thus, given the same spectrum, amplitude noise is less destructive than phase noise because of reduced spectral density at the second harmonic of the synchrotron frequency. The small amplitude synchrotron frequency of the SSC collider rings, about $4.2 \mathrm{~Hz}$, is such that the first synchrotron sideband is close enough to the carrier that the noise power there could be troublesome. In order to provide a basis for establishing design criteria, we have investigated this in some detail. ${ }^{25}$ As an illustration of the DKW theory we present some of those results here.

When establishing design criteria, one important quantity to consider is the longitudinal emittance, which is a measure of longitudinal bunch size. The conventional way to define the longitudinal emittance is through the phase space area containing a given fraction of the beam bunch. Thus we can define the $f$-emittance, $J_{f}(t)$, by

$$
\int_{0}^{J_{f}(t)} \rho_{c}(J, t) d J=f
$$


where $\rho_{c}(J, t)=\rho(J, t) / \int_{0}^{J_{b}} \rho(J, t) d J$ and $f$ is the fraction of particles under consideration. The emittance-doubling time, $t_{d}$, is then defined by

$$
J_{f}\left(t_{d}\right)=2 J_{f}(0) .
$$

Here we assume that $J_{f}(t)$ is monotonically increasing with $t$. Another measure of the longitudinal bunch size is the mean of $J(t)$ conditioned on $J(t)<J_{b}$; that is,

$$
\bar{J}(t):=E\left[J(t) \mid J(t)<J_{b}\right]=\int_{0}^{J_{b}} J \rho_{c}(J, t) d J .
$$

Before we discuss our numerical calculations of emittance-doubling time, we give a first-principles derivation of a simple approximation to $\bar{J}(t)$ that was used in the $\mathrm{CDR}^{12}$ to determine noise levels corresponding to a 50 -h emittance-doubling time. To our knowledge, a comparable treatment has not appeared in the literature.

Differentiating Equation (4.3) and using Equations (2.4) and (2.5b) yields

$$
\frac{d \bar{J}(t)}{d t}=\int_{0}^{J_{b}} D^{\prime}(J) \rho_{c}(J, t) d J+\left(J_{b}-\bar{J}(t)\right) D\left(J_{b}\right) \frac{\partial \rho_{c}}{\partial J}\left(J_{b}, t\right) .
$$

In the white-noise, small-oscillation case $\left(U(\phi)=\frac{1}{2} \Omega^{2} \phi^{2}\right)$,

$$
D(J)=\frac{1}{2} \Omega^{2}\left(\Omega S_{\psi} J+S_{a} \frac{1}{2} J^{2}\right),
$$

which gives

$$
\frac{d \bar{J}}{d t}=\frac{1}{2} \Omega^{2}\left(\Omega S_{\psi}+S_{a} \bar{J}\right)+W\left(t, J_{b}\right)
$$

where $W\left(t, J_{b}\right)$ is the second term on the right-hand side of Equation (4.4). Equation (4.5) can be derived directly from Equation (2.21) or in the small $k$ asymptotics for Equation (3.1). If we ignore $W$ and define $X=\bar{J} / \Omega$, then Equation (4.6) is exactly Equation (4.4-21) of the CDR: ${ }^{12}$

$$
\frac{d X}{d t}=\frac{1}{2} \Omega^{2}\left(S_{\psi}+S_{a} X\right) .
$$

The doubling time of the mean is now easily calculated. For amplitude noise,

$$
t_{d}=\frac{2 \ln 2}{\Omega^{2} S_{a}}
$$

and for phase noise,

$$
t_{d}=\frac{2 \bar{J}_{0}}{\Omega^{3} S_{\psi}} .
$$

Using Equation (3.4), $\bar{J}_{0} \approx \mu=3.937 \mathrm{rad} \mathrm{sec}^{-1}$ for the SSC parameters. If we use $t_{d}=50 \mathrm{~h}$, then $S_{\psi}=2.32 \times 10^{-9} \mathrm{rad}^{2} \mathrm{~Hz}^{-1}$ and $S_{a}=10.9 \times 10^{-9} \mathrm{~Hz}^{-1}$. With these white-noise spectral densities we then computed the diffusion coefficients 


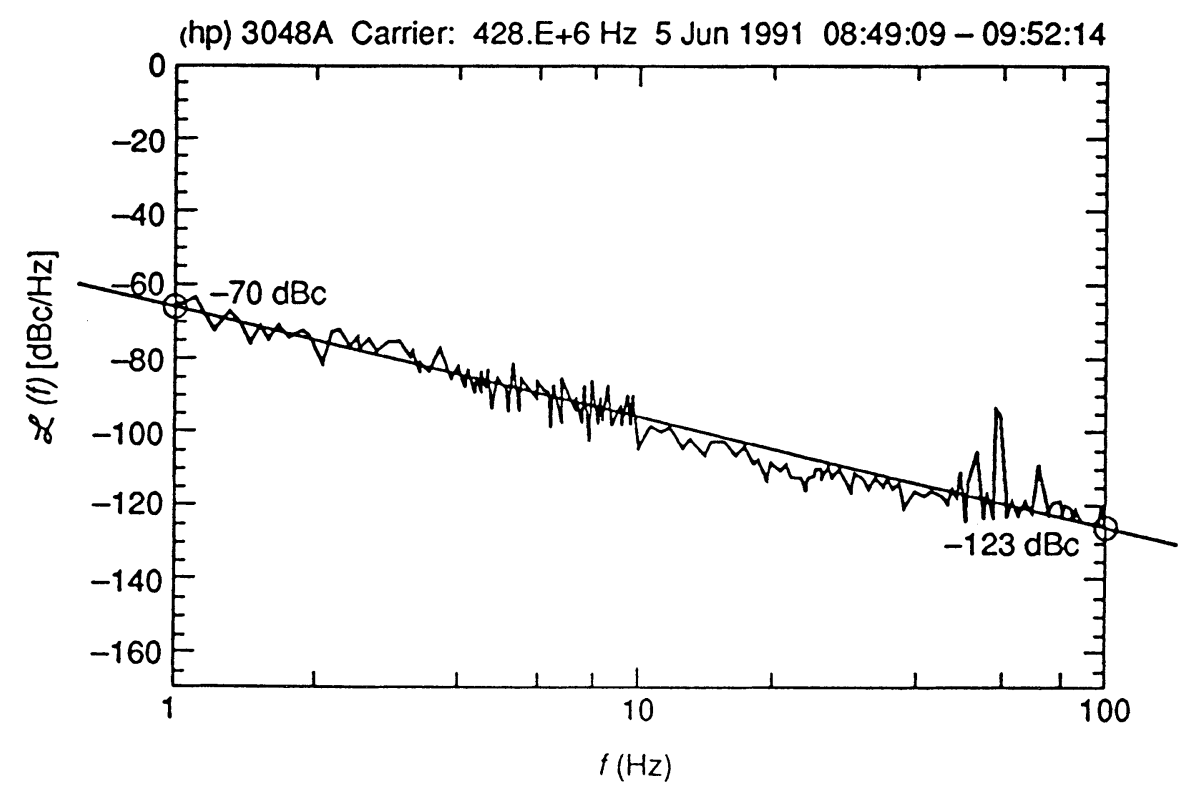

FIGURE 7: Phase Noise Spectral Density Measured on an HP8662 Synthesizer. The straight line is the fit, Equation (4.10), to the spectrum.

$D(J)$ according to Equation (3.1) and integrated the diffusion Equation (2.4) using the method of lines. The numerical results of $\rho(J, t)$ were then used to calculate the mean emittance defined by Equation (4.3) as a function of time. We found an emittancedoubling time of $80 \mathrm{~h}$ for white amplitude noise and $59 \mathrm{~h}$ for white phase noise. Thus the simple approximation gives more conservative results, i.e., lower spectral density for the same emittance-doubling time. This is expected because we obtained the simple approximation by neglecting the second term $W\left(t, J_{b}\right)$ in Equation (4.6), which is always negative under the condition that $\bar{J}$ is less than $J_{b}$, the action at the absorbing boundary.

The phase noise for a synthesizer of a type being considered for use in the SSC rf system is shown in Figure 7. A reasonable fit to the spectrum is given by

$$
S_{\psi}(\omega)=\left\{\begin{array}{lll}
1.3 \times 10^{-5} / \omega^{2.65} & \omega<628.3 & \mathrm{rad} \mathrm{sec}^{-1} \\
0.5 \times 10^{-12} & \omega \geq 628.3 & \mathrm{rad} \mathrm{sec} \\
\end{array}\right.
$$

The diffusion coefficient is shown in Figure 8; surprisingly, it is nearly linear, as the dashed line indicates. Figure 9 shows $\rho(J, t)$ vs. $J$ for various $t$, and Figure 10 shows $\vec{J}(t)$ and $J_{f}(t)$ for two values of $f$. The doubling times of the mean, $39 \%$ and $95 \%$ emittances, are $\sim 55 \mathrm{~h}$, which is on the order of the CDR design criterion. 


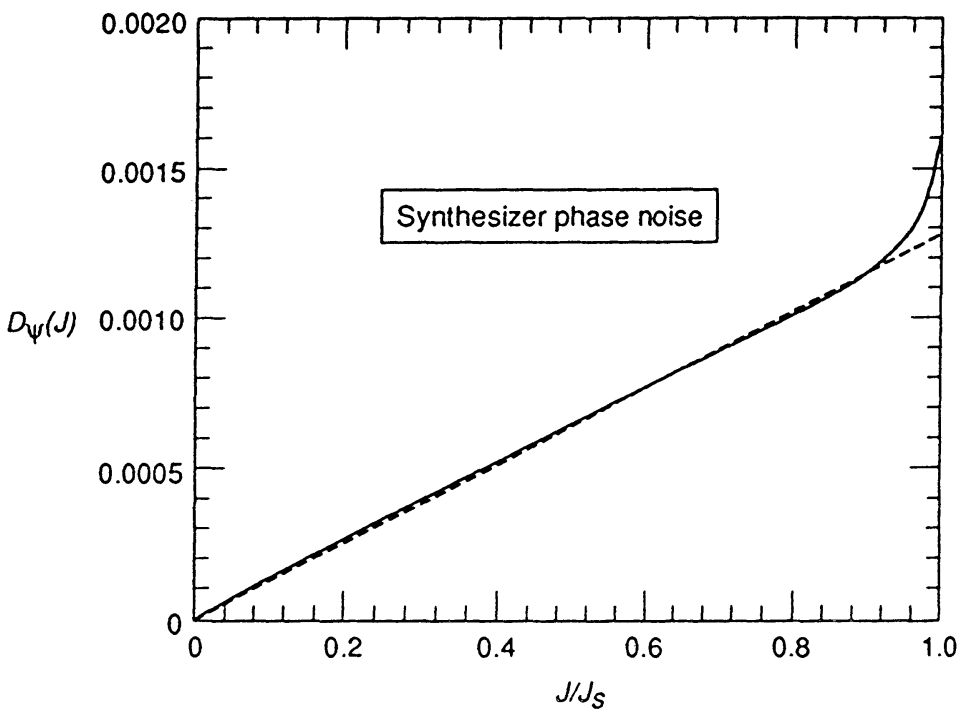

FIGURE 8: Diffusion Coefficient for Spectral Density of Figure 7, Using Equation (4.10). Dashed line is a linear approximation.

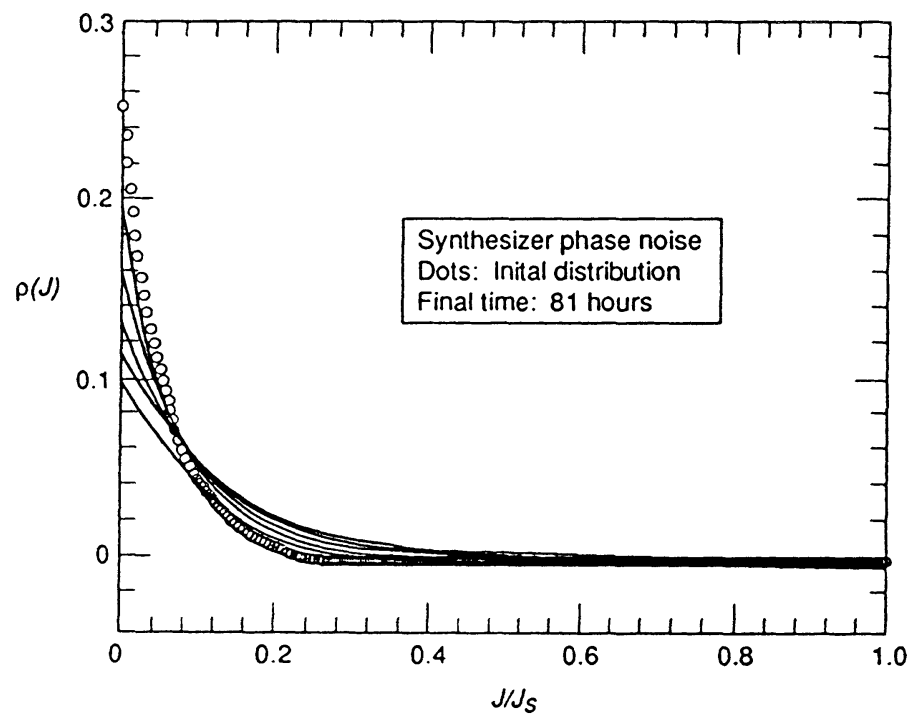

FIGURE 9: Evolution of Action Density for Case of Figure 8 in Increments of $81 / 5 \mathrm{~h}$. The initial density is given by circles; final density is at $81 \mathrm{~h}$. 


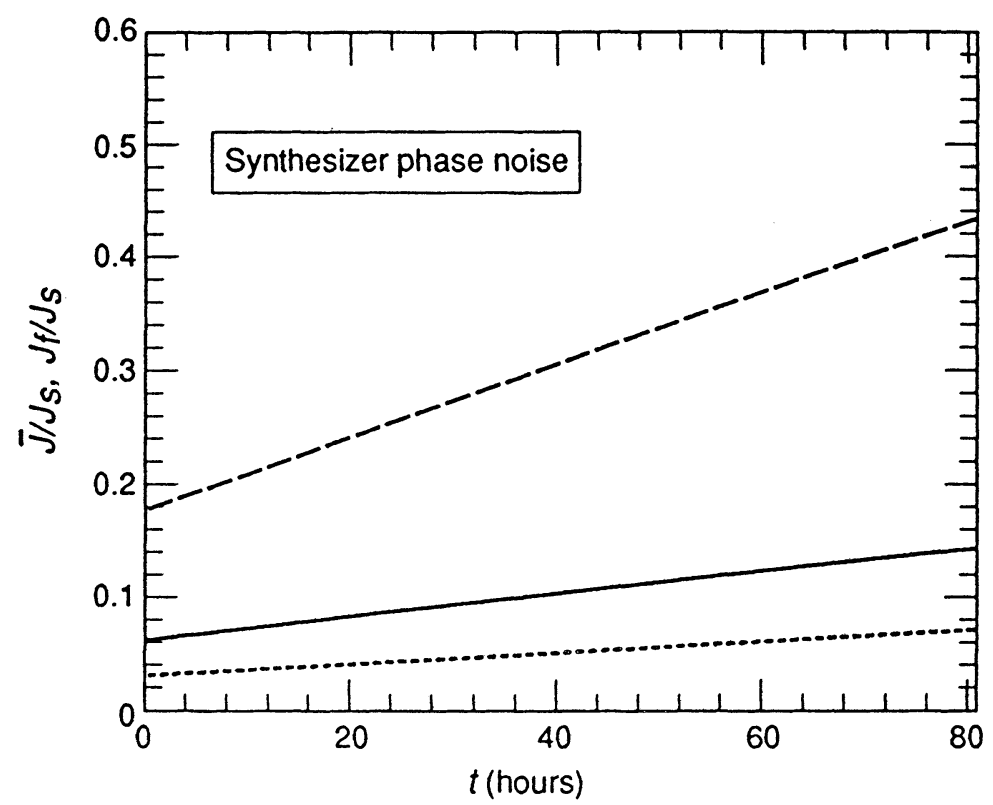

FIGURE 10: Longitudinal Emittance Growth for Case of Figure 8. Solid: mean emittance. Dashed: $95 \%$ emittance. Dotted: $39 \%$ emittance.

Some freedom in the choice of rf frequency makes it of interest to understand the dependence of the diffusion on $\omega_{r f}=2 \pi h / T_{0}$. This can be done to some extent in the white noise case. Since $\Omega^{2}=\omega_{r f}|\eta| e V / p_{s} C_{0}, \Omega^{2} \propto \omega_{r f}$ for a fixed $V$. If we let $P=\Omega \tilde{P}$, then from Equation (2.3b) the $(\tilde{P}, \phi)$ phase space is independent of $\Omega$ (and $\left.\omega_{r f}\right)$, and $2 \pi J=\oint P d \phi=\Omega \oint \tilde{P} d \phi=: \Omega 2 \pi \tilde{J}$, where the last equality defines $\tilde{J}$. Let $p(\tilde{J}, t)$ be the $\tilde{J}$ density; then $p(\tilde{J}, t)=\Omega \rho(J, t)$, and Equation (2.4) becomes

$$
\frac{\partial p}{\partial t}=\frac{\partial}{\partial \tilde{J}}\left(\frac{D(\Omega \tilde{J})}{\Omega^{2}} \frac{\partial p}{\partial \tilde{J}}\right) .
$$

From Equations (2.7) and (2.8), $\phi_{m}=\phi_{m}(J, \Omega)$ is defined by

$$
2 \pi J=4 \sqrt{2} \Omega \int_{0}^{\phi_{m}} \sqrt{\cos \phi-\cos \phi_{m}} d \phi .
$$

Since $J=\Omega \tilde{J}$, it is clear from (4.12) that $\phi_{m}(\Omega \tilde{J}, \Omega)$ is independent of $\Omega$, and it follows from Equation (2.21) that for white noise

$$
D(\Omega \tilde{J})=\Omega^{4} \mathcal{D}(\tilde{J}),
$$

where $\mathcal{D}(\tilde{J})$ is independent of $\Omega$. Letting $\tau=\Omega^{2}$ t, we obtain the initial-boundary value problem for $p(\tilde{J}, t)$ as 


$$
\begin{aligned}
& \frac{\partial p}{\partial \tau}=\frac{\partial}{\partial \tilde{J}} \mathcal{D}(\tilde{J}) \frac{\partial p}{\partial \tilde{J}} \\
& p(\tilde{J}, 0)=\Omega \rho_{0}(\Omega \tilde{J}) \\
& p\left(\tilde{J}_{b}, \tau\right)=0 .
\end{aligned}
$$

The $\Omega$ dependence now enters only in two ways: (1) through the initial density, Equation (4.14b), and (2) through the time scaling. If the initial action density $\rho_{0}(J)$ is given by (3.4), then $\bar{J}_{0}=\mu=\omega_{r f}^{2} \epsilon_{L}|\eta| / p_{s} \nu_{s}$ where $\epsilon_{L}=\sigma_{\delta E} \sigma_{\delta t}$ and $\delta E$ and $\delta t$ are the energy and time deviations from the synchronous particle, respectively. Thus, if $\epsilon_{L}$ is fixed, and recalling that $\Omega^{2} \propto \omega_{r f}$ for a fixed $V$, we see that increasing $\omega_{r f}$ broadens the initial distribution. If we assume that a broader initial distribution leads to a faster deterioration of the beam then

$$
\tau_{c 2}<\tau_{c 1} \text { when } \omega_{r f 2}>\omega_{r f 1},
$$

where $\tau_{c}$ is a critical scaled time for loss of beam quality. Therefore, (4.15) reads $\Omega_{2}^{2} t_{c 2}<\Omega_{1}^{2} t_{c 1}$ and

$$
t_{c 2}<\frac{\omega_{r f 1}}{\omega_{r f 2}} t_{c 1} .
$$

If $f_{r f 1}=360 \mathrm{MHz}$ and $f_{r f 2}=480 \mathrm{MHz}$, two of the values that have been considered at the SSC, then we expect $t_{c 2}<\frac{3}{4} t_{c 1}$ because we have a broader initial beam in case 2. This is consistent with Figure 11, where we plot the relative emittance as a function of time for phase noise of $S_{\psi}=2.32 \times 10^{-9} \mathrm{rad}^{2} \mathrm{~Hz}^{-1}$ and an initial emittance of $\epsilon_{L}=0.233 \mathrm{eV}$-sec. For example, for a relative emittance of 0.15 , we find $t_{c 2}=23$ $\mathrm{h}$ and $t_{c 1}=73 \mathrm{~h}$. Our calculations show that the mean emittance-doubling time in case 1 is $86 \mathrm{~h}$, and that it increases to $142 \mathrm{~h}$ in case 2 , in contrast to the expectation in Equation (4.16); see Figure 12. To understand this, we note that the narrower beam has steeper gradients; thus the diffusion process works faster, giving a shorter doubling time even though the resulting beam is still relatively narrow and could be narrower than the initial beam for the larger $\omega_{r f}$. This points out that emittance-doubling times may not be an appropriate design criterion. A more appropriate criterion may be the time it takes for the beam to reach a certain critical size relative to the bucket area.

\subsection{Noise in Superslow Extraction}

As described in more detail elsewhere, ${ }^{15,17,19}$ the presently envisioned extraction geometry at the SSC has a slablike single crystal lying in the midline of the beampipe

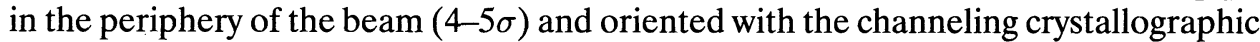
planes horizontal. Because the crystal is bent vertically, the protons that reach it and are channeled will be deflected vertically. The subsequent vertical drift leads them to enter a field-free region and get extracted into an external beamline. 


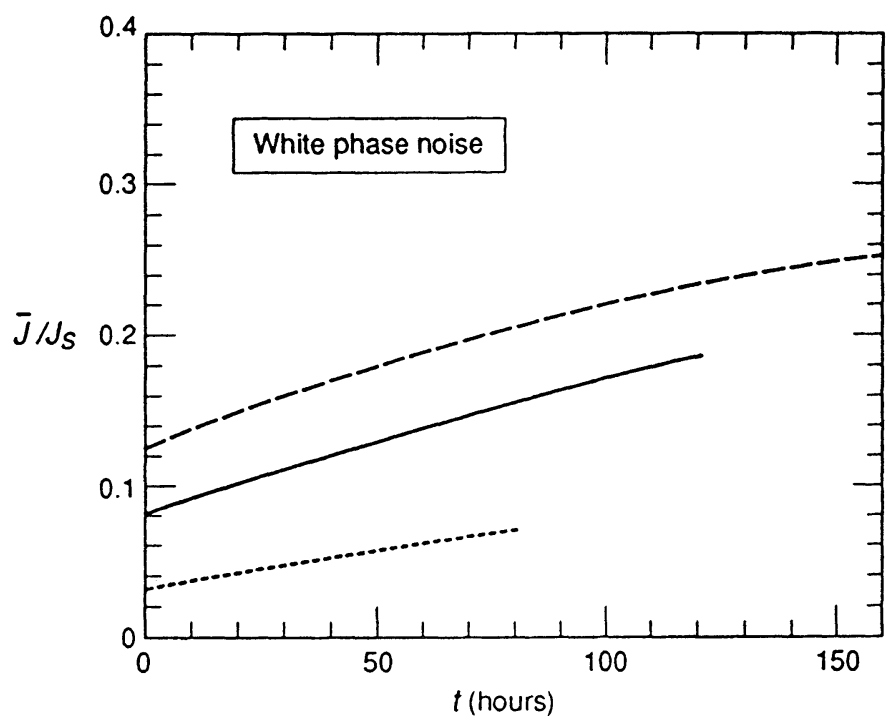

FIGURE 11: Dependence of Longitudinal Emittance Growth on the rf Frequency for White Phase Noise with $S_{\psi}=2.324 \times 10^{-9} \mathrm{rad}^{2} \mathrm{~Hz}^{-1}$. Dashed: $480 \mathrm{MHz}$. Solid: $360 \mathrm{MHz}$. Dotted: $180 \mathrm{MHz}$. Here $J=$ $\frac{1}{2 \pi} \oint P d \phi$.

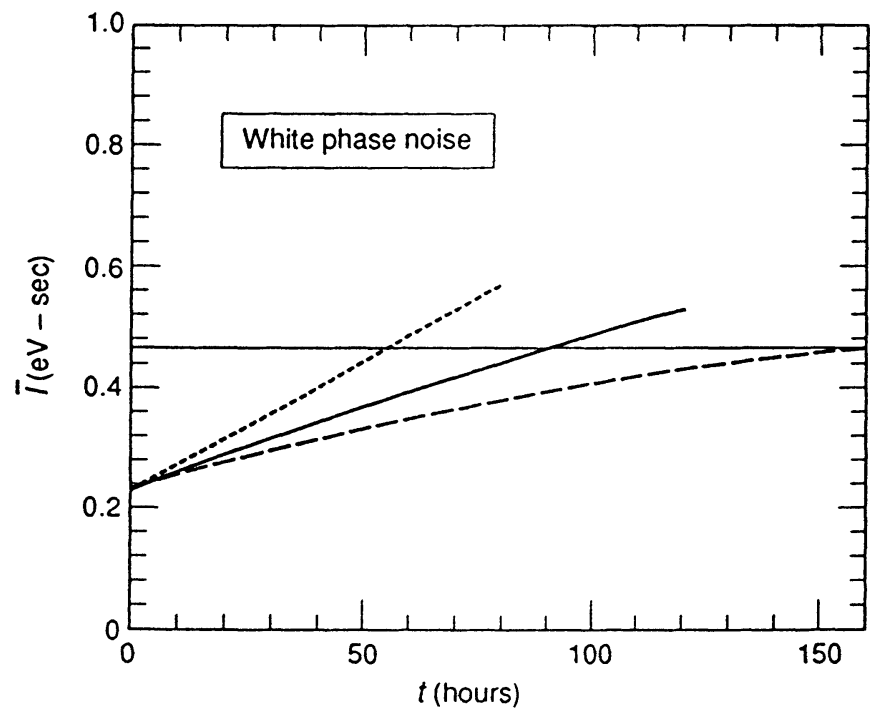

FIGURE 12: Same as Figure 11, except $I=\frac{1}{2 \pi} \oint \delta E d(\delta t)$. Note that $J=\left(\frac{\omega_{r f}^{2}|\eta|}{p_{s^{\nu}}{ }^{\nu}}\right) I$. 
One possibility that has been investigated ${ }^{15,17,19}$ for feeding protons onto the crystal is to locate the crystal in a region where the dispersion is large and then feed particles by manipulating the longitudinal degree of freedom. Several possibilities for manipulating the longitudinal degree of freedom have been suggested; we discuss below the results obtained by injecting noise into the rf system. While we discuss both phase and amplitude noise, the emphasis will be on the latter. Many of our results on phase noise have appeared before, ${ }^{15,16}$ so they will only be summarized here. Initially, we had concentrated on phase noise because of some concern with the operating state of the final rf amplifiers in the collider. If the amplifiers were to be operated in saturation, control of amplitude noise might be unreliable. However, as this is not to be the case ${ }^{26}$ amplitude noise is a viable option.

The first issue that arises is whether the feed rates onto the crystal are adequate to supply the beam at the desired rate. (For the proposed SFT experiment, the desired extraction rate is $2 \times 10^{8}$ particles per second.) This is simple to ascertain, and our Monte Carlo simulations have extraction rates that are considerably larger than necessary. Indeed, to obtain adequate statistics with the number of tracks (1000) we use, the noise variances in the simulations are larger than would be used in the actual physical system. One must extrapolate to find the spectral density that would give the desired extraction rate. Relative extraction rates are shown as a function of the noise standard deviation in Figures 13(a) and 13(b) for low-pass amplitude and phase noise, respectively, and in Figure 13(c) for white amplitude noise.

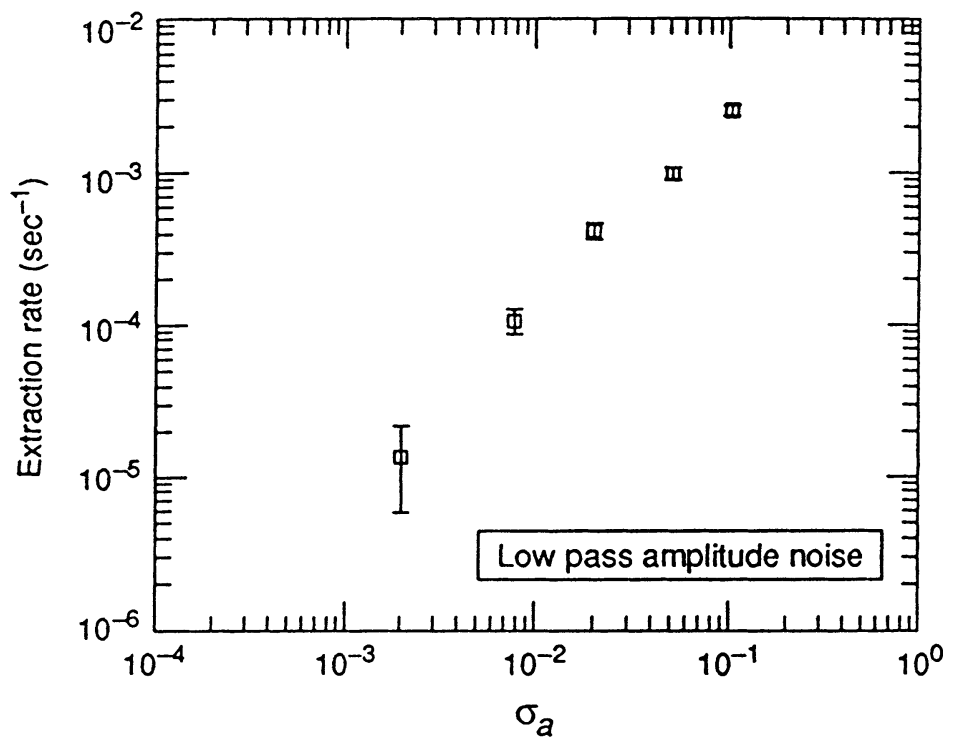

FIGURE 13a: 


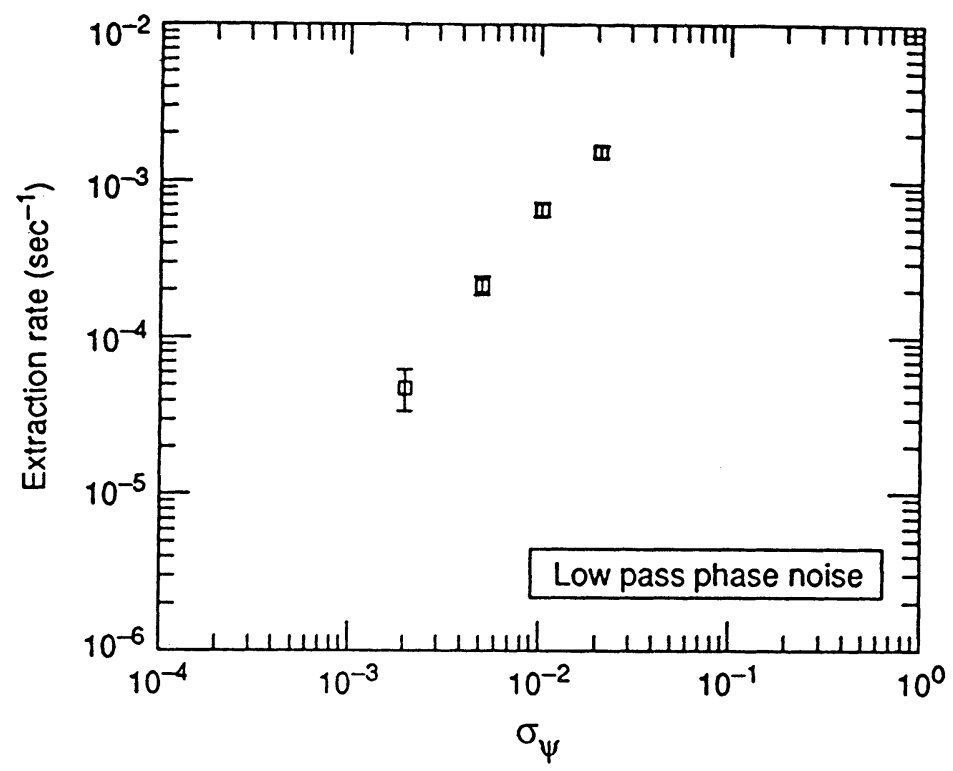

FIGURE 13b:

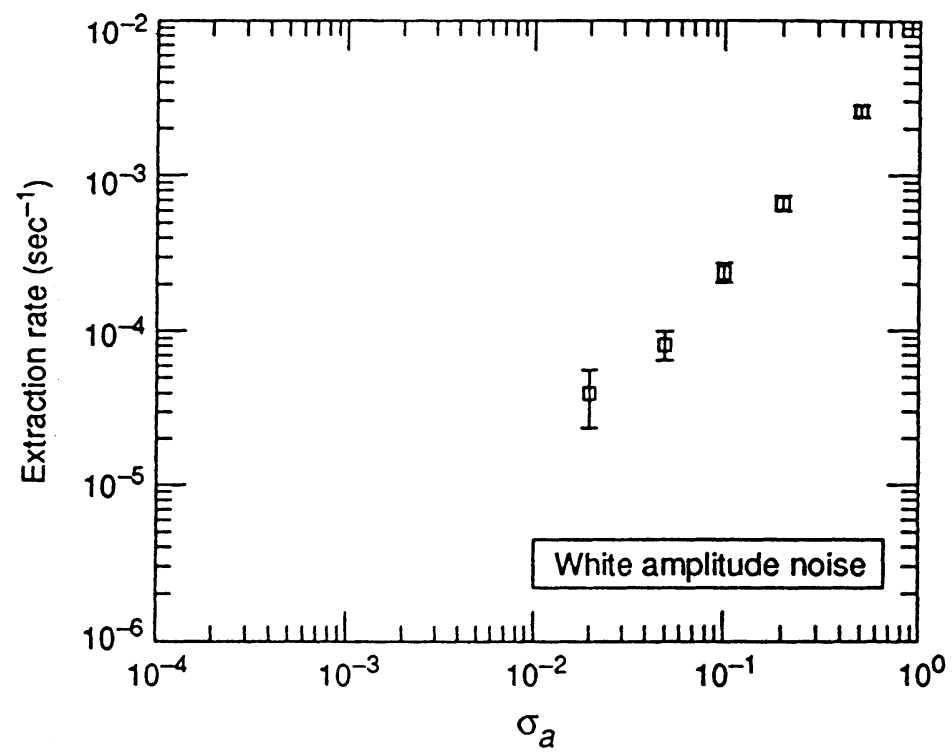

FIGURE 13c:

FIGURE 13: Normalized Extraction Rate as a Function of Noise Standard Deviation. (a) Low-pass amplitude noise. (b) Low-pass phase noise. (c) White amplitude noise. 
For the last example, the rms noise amplitude at the SFT extraction rate is approximately $400 \mathrm{kV}$ in a $20-\mathrm{MV}$ system. In determining the extraction rate, we have disregarded the hits from the initial transient, which occur because some of the tracks $(\approx 30)$ have initial conditions such that, over the first synchrotron period, they are brought onto the crystal. Obviously, they hit the crystal even in the absence of noise. We can also use the numerical solution of the diffusion equation with realistic values of the diffusion coefficient to estimate the flux onto the crystal. In reality, the absorbing boundary is defined by the inner edge of the crystal instead of a particular action near the separatrix as has been used in previous discussions. Thus one must also consider the betatron motion in determining the flux onto the crystal. The effect of the betatron motion on the extraction process will be discussed below. Extension of the DKW diffusion theory, which is strictly longitudinal, to include the betatron motion has been made by the authors ${ }^{27}$ and shows excellent agreement with tracking simulations.

Of greater concern than the extraction rate is the issue of the effect of noise on the longitudinal core of the beam. It is undesirable to produce a beam with a broad distribution. Obtaining the extraction rates by uniformly filling the tail at the expense of small-amplitude particles is to be avoided in order to preserve the beam bunchto-bucket ratio. It is immediately clear from Figure 5(a) that white phase noise is not suitable. This is also seen in Figure 14(a), the scatter plot of initial conditions of those particles that eventually strike the crystal. However, by filtering the phase noise to eliminate the frequency components near the small-amplitude synchrotron frequency, the situation changes dramatically. This is shown in the scatter plot in Figure 14(b) for the low-pass case. A similar result is obtained in the notched case. The hole in the phase space for core particles is obvious and consistent with the behavior of the diffusion coefficient discussed in Section 2. Amplitude noise is already at an advantage in this regard-even in the case of white noise-because, as discussed in Section 2 , the diffusion coefficient for white amplitude noise is proportional to $J^{2}$ for small $J$. The scatter plot for this case is shown in Figure 15(a). Recall from the discussion in Section 2 that filtering a small band of frequencies around $2 \Omega$ reduces the diffusion coefficient for small $J$ even further. The scatter plot for this case is shown in Figure 15(b). In the case of amplitude noise the longitudinal emittance growth is small. This is seen in Figure 4(b) (upper curve), where the fluxes are considerably larger than SFT requires. Including the effect of the betatron motion, discussed further below, we see in Figure 4(b) (lower curve) that the emittance is remarkably constant. These results demonstrate that we can protect the beam core from excessive degradation by simple manipulation of the noise spectrum from the generator. In principle, an arbitrary degree of reduction can be achieved by filtering higher and higher harmonics of $\Omega$. Indeed, filtering the noise with a low-pass filter gives a diffusion coefficient that vanishes below a value of action at which the amplitude-dependent synchrotron frequency, $\omega_{s}(J)$, equals the cutoff frequency of the filter.

The issue of how the halo particles that strike the crystal are distributed in the edge of the crystal is also of some concern. For any given particle that is about to be extracted, the extraction process is discrete. As it passes by the crystal on each turn around the machine, the particle is simultaneously moving around its betatron ellipses while its closed orbit is moving horizontally, carried by the longitudinal motion on the 


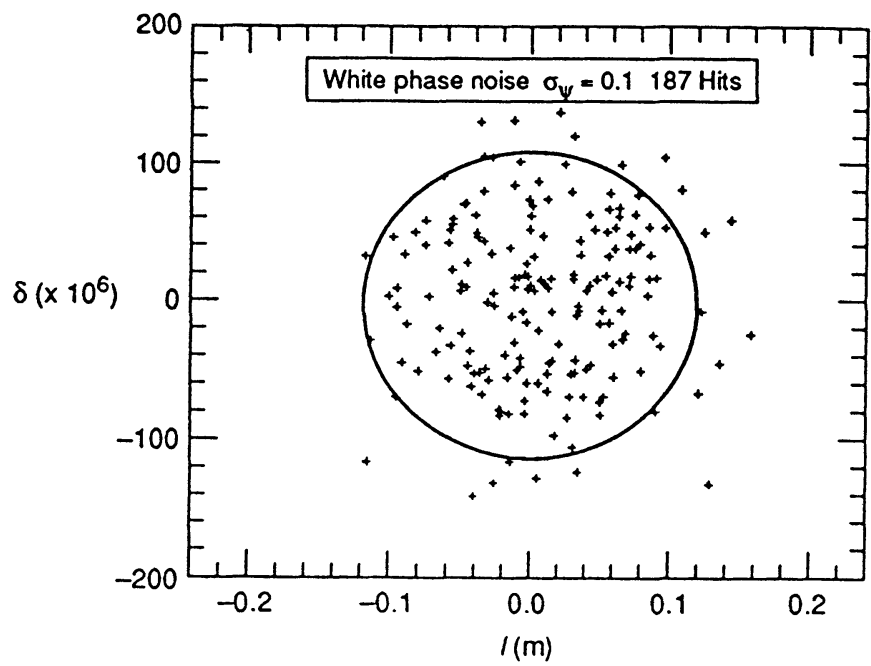

FIGURE 14a:

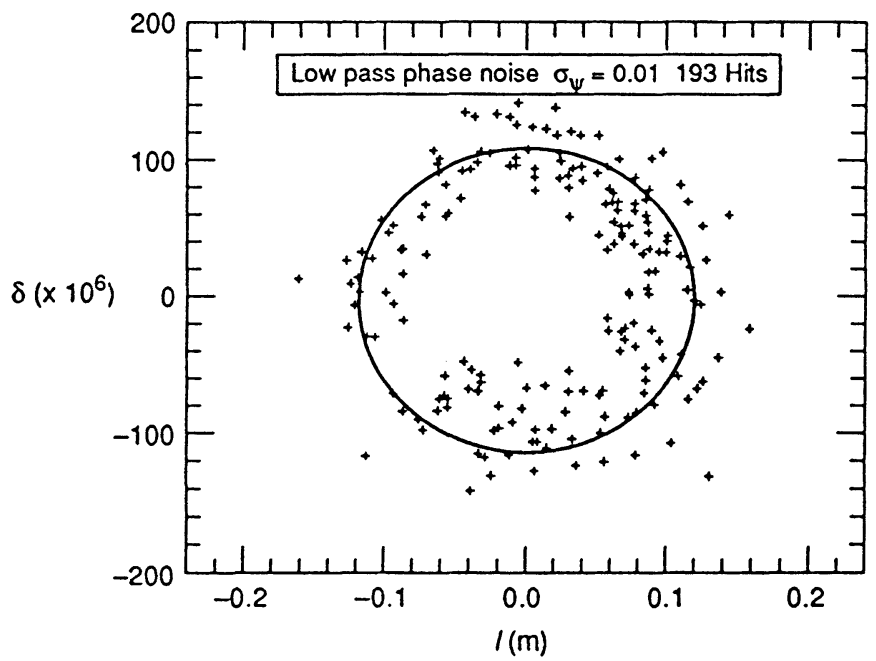

FIGURE 14b:

FIGURE 14: Initial Distribution in Longitudinal Phase Space of the Protons that Strike the Bent Crystal in $10^{6}$ turns as a Result of Injecting Phase Noise. (a) White phase noise with $\sigma_{\psi}=0.1$. (b) Low-pass phase noise with $\sigma_{\psi}=0.01$. The number of hits is 187 in case (a) and 193 in case (b). The circle is the orbit whose synchrotron frequency is $0.95 \Omega$. 


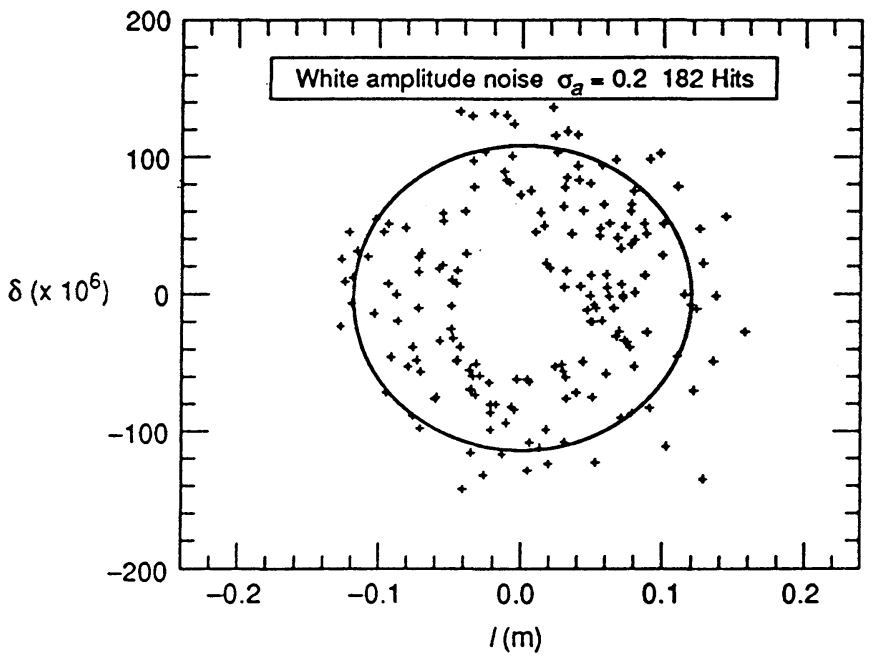

FIGURE 15a:

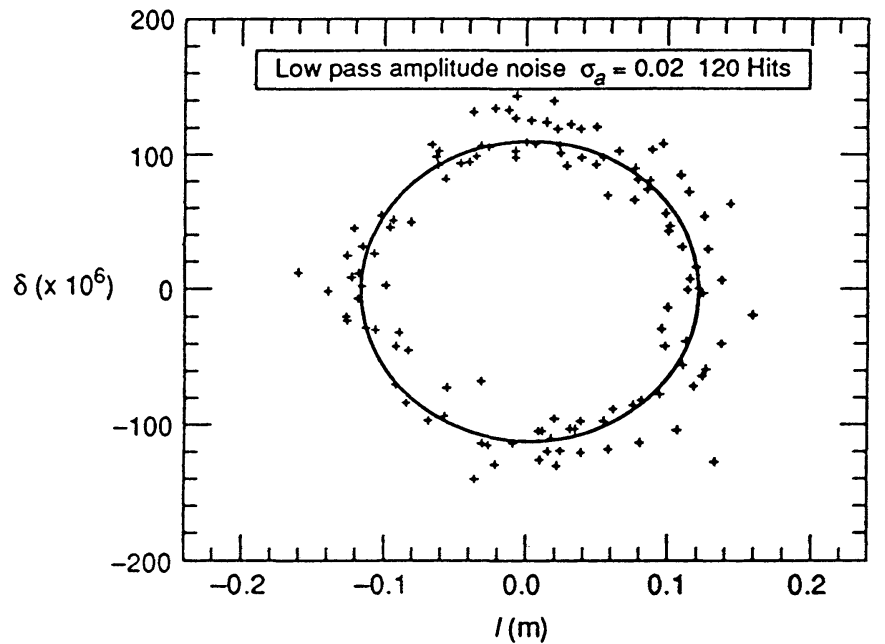

FIGURE 15b:

FIGURE 15: As in Figure 14 for Amplitude Noise. (a) White noise with $\sigma_{a}=0.2$ and 182 hits. (b) Low-pass noise with $\sigma_{a}=0.02$ and 120 hits. 


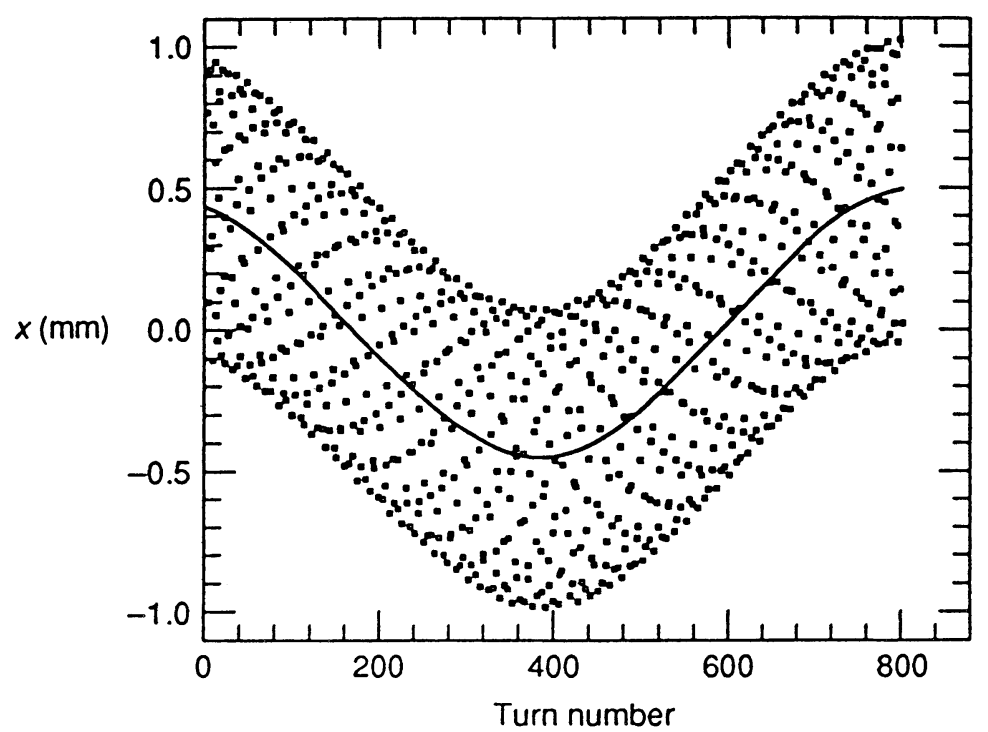

FIGURE 16: Coordinate $x$ of a Particle as a Function of Turn Number at the Location of Bent Crystal. The initial condition of the particle is such that it strikes the bent crystal in less than one synchrotron period. The curve is the closed orbit defined by the synchrotron motion.

synchrotron ellipse. (Recall that the crystal is located at a point of high dispersion in the lattice.) On this time scale, the diffusion is negligible; in fact, such a description does not apply. Even though the superposition of the betatron amplitude and the closed orbit position may extend past the crystal edge, the time of extraction depends on the betatron phase of the particle when it arrives in the vicinity of the crystal. On a long time scale, diffusion gives rise to an increase in the closed orbit amplitude; this is the process by which the particle reaches the vicinity of the crystal in the first place.

The first two effects, the superposition of the closed orbit and betatron motion, can be seen in Figure 16. This is for a particle whose initial conditions, drawn from distributions of $\delta$ and $x_{\beta}$, already bring it to the crystal in a synchrotron period or less. Here the particle has struck the crystal in about 800 turns, when the superposition of its closed orbit position and betatron envelope has reached the crystal edge. For a particle for which the closed orbit diffusion is meaningful, the three effects together continuously feed particles onto the crystal. Together these give rise to a distribution of strikes on the crystal within a layer of thickness $\approx 10 \mu \mathrm{m}$. The thickness of the layer depends on the betatron tune, and the detailed distribution also depends on the value of $\beta_{x}$ at the crystal. ${ }^{15}$ We show such distributions in Figures 17(a) and 17(b). While polishing and etching techniques are available that provide good crystal at the edge, the distribution of the extracted particles could have implications for local heating 


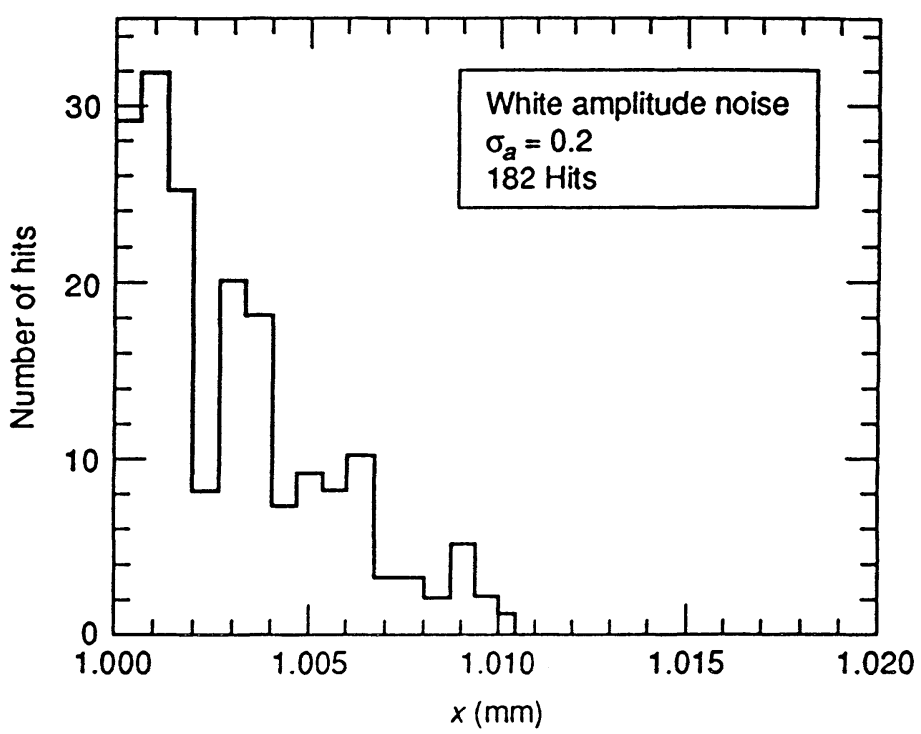

FIGURE 17a:

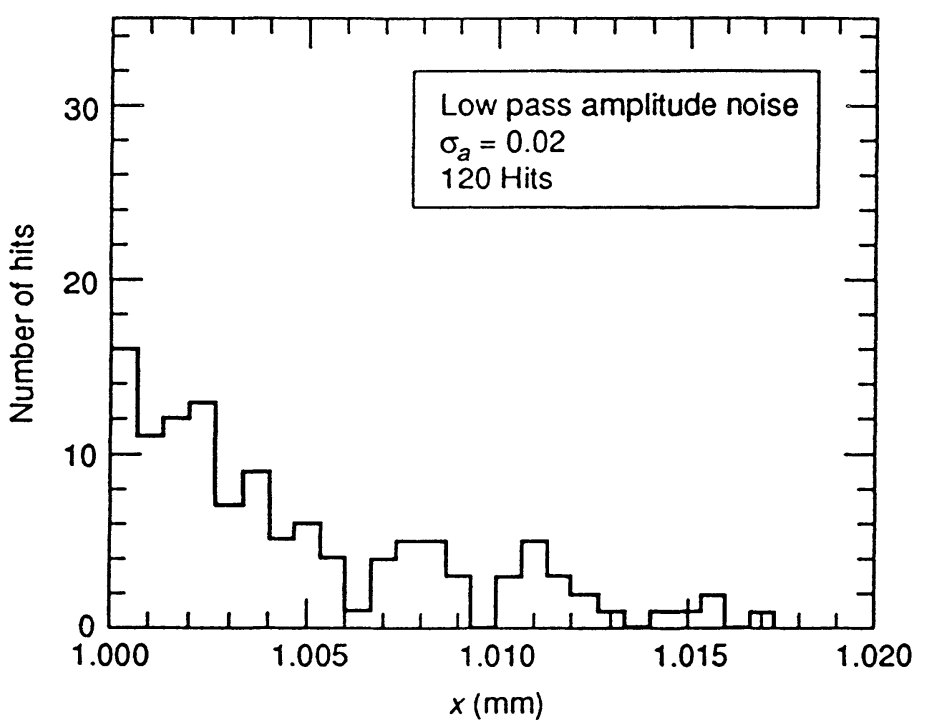

FIGURE 17b:

FIGURE 17: Distribution of Hits Across the Bent Crystal as a Result of Injecting Amplitude Noise. (a) White noise with $\sigma_{a}=0.2$. (b) Low-pass noise with $\sigma_{a}=0.02$. 
and radiation damage of the crystal. Such potential damage must be considered in the design of the extraction apparatus. Having as uniform a distribution as possible is obviously desirable. Some ideas by which some control over this distribution may be obtained have been suggested. ${ }^{28}$ This particular issue is still in a preliminary state of investigation and is in the realm of future work.

\section{CONCLUSIONS}

In this paper, we have considered the diffusion of particles in longitudinal phase space in a proton synchrotron due to noise in the rf system. The theory, which was reviewed in Section 2, is essentially that of Dôme and Krinsky and Wang; however, we have extended it and discussed it in a way that enables it to serve as a prelude to our detailed investigation in terms of stochastic limit theorems. ${ }^{6}$ We have developed an accurate and efficient algorithm for numerically integrating the diffusion equation, Equation (2.4), for general diffusion coefficients and general initial densities, and thus we have confidence in our numerical results. We have made extensive comparisons between the DKW diffusion theory and tracking simulations. The agreement is good, and thus we have confidence that the theory is valid in the parameter region of the SSC; furthermore, the calculations based on the theory are much faster than the tracking simulation. The theory has been applied to two problems: (1) the growth of longitudinal emittance due to intrinsic sources of noise in the synchrotron rf system (herein specifically pertaining to the SSC); and (2) the controlled diffusion of particles to the edge of the rf bucket in order to extract them from the machine to feed a fixedtarget physics experiment or to provide test beams.

We have considered several sources of noise in the rf system of the collider rings at the SSC and have estimated their effect on the longitudinal emittance of the beam. Methods to mitigate against noise-feedback loops, for example-have not been investigated here, although it would be straightforward to do so. The low synchrotron frequency of the collider has caused the issue of noise in the rf to be of some concern. Looking only at noise in the low-level rf signal source, we have found that emittance growth rates are comparable with design specifications. Of course, as other sources of noise are identified, they should be investigated as well. From the theory of diffusion in action in Section 2, we have derived a simple approximation for the time evolution of the longitudinal emittance. This approximation, essentially arising from scaling arguments, has been in rather general use in the accelerator physics community for several years. Our derivation shows the conditions that must be satisfied for it to be valid. Roughly, the flux across the bucket boundary must be small. Lastly, we have explored the effect of changing the frequency of the collider rf system on the growth of the longitudinal emittance for given conditions of the injected beam.

In contrast to the undesirable but unavoidable slow increase of the longitudinal emittance from intrinsic noise sources is the deliberate injection of noise into the rf system to feed an extraction line. In order to operate a beamline in tandem with collider operations, it is crucial to show that this can be done without significantly making the bunches too long. In Section 4.2 we have shown that by appropriate manipulation 
of the noise spectrum, adequate extraction rates can be obtained without significantly increasing the longitudinal emittance. Indeed, in some cases, when the effect of the betatron oscillations is accounted for in the extraction process, the longitudinal emittance has been found to be essentially constant. There seems to be no fundamental difficulty in applying this technique to ultraslow extraction at the SSC. Certain details of the extraction process, especially pertaining to the bent crystal extraction septum, are the subject of ongoing research. An experiment (E853) is now being carried out on the Tevatron to test this extraction scheme.

\section{ACKNOWLEDGEMENTS}

The support of this work by Michael Syphers is gratefully acknowledged. B.S.N. has been supported in part by the Texas National Research Laboratory Commission under Grant RGFY93-321, and J.A.E. has been supported by the SSC Laboratory under the visiting scientist program.

\section{REFERENCES}

1. D. Boussard, L. Evans, J. Gareyte, T. Linnecar, W. Mills, and E.J.N. Wilson, "Acceleration and storage of a dense single bunch in the CERN SPS," IEEE Trans. Nucl. Sci. NS-26, 3484 (1979).

2. D. Boussard, G. Dôme, and G. Graziani, "The influence of rf noise on the lifetime of bunched proton beams," in Proceedings of the 11th International Conference on High-Energy Accelerators, W.S. Newman, ed. (Birkhauser, Basel, 1980), p. 620.

3. D. Boussard, "RF techniques for $p \bar{p}$," in CERN Accelerator School on Antiprotons for Colliding Beam Facilities, CERN Report No. 84-15, 1984, pp. 261-290.

4. G. Dôme, "Diffusion due to rf noise," in CERN Advanced Accelerator School on Advanced Accelerator Physics, CERN Report No. 87-03, 1987, pp. 370-401.

5. S. Krinsky and J.M. Wang, "Bunch diffusion due to rf noise," Part Accel. 12, 107 (1982).

6. R. Cogburn, J.A. Ellison, B.S. Newberger, and H.-J. Shih, in preparation.

7. J.A. Ellison and T. Guinn, "Statistical equilibrium, planar channeling, and the continuum model," Phys. Rev. B13, 1880 (1976).

8. C.W. Gardiner, Handbook of Stochastic Methods, second edition (Springer-Verlag, 1990); T.C. Gard, Introduction to Stochastic Differential Equations (Marcel Dekker, Inc., 1988).

9. R. Cogburn and J.A. Ellison, "A stochastic theory of adiabatic invariance," Comm. Math. Phys. 148 (1992), pp. 97-126.

10. H.-J. Shih, J.A. Ellison, and W.E. Schiesser, "Reliability of numerical solutions of a diffusion equation modeling rf noise-induced dilution in particle beams," in Advances in Computer Methods for Partial Differential Equations VII, R. Vichnevetsky, D. Knight and G. Richter, eds. (IMACS, New Brunswick, 1992), p. 663.

11. L.H. Koopmans, The Spectral Analysis of Time Series (Academic Press, 1974).

12. SSC Central Design Group, Superconducting Super Collider Conceptual Design Report, SSC-SR-2020 (March 1986), p. 162; S. Chattopadhyay, "RF noise," SSC Laboratory Report SSC-N-38 (August 1985).

13. E.N. Tsyganov, "Estimates of cooling and bending processes for charged particle penetration through a monocrystal," Fermilab Report TM-684 (September 8, 1976). 
14. B. Cox, J. Lach, M. Maslov, N. Mokhov, C.T. Murphy, and R.J. Stefanski, "The possibility of parasitic $20 \mathrm{TeV}$ beams extracted from the SSC using bent crystals," in Proceedings of the Summer Study on High Energy Physics, S. Jensen, ed. (World Scientific, Singapore, 1989), p. 536.

15. B.S. Newberger, H.-J. Shih, and J.A. Ellison, "Super slow extraction at the SSC Using Channeling in a Curved Crystal," Nucl. Inst. Meth., A325, 9 (1993).

16. J.A. Ellison, B.S. Newberger, and H.-J. Shih, "Effect of rf phase noise on the SSC beam," in Conference Record of the 1991 IEEE Particle Accelerator Conference, Institute of Electrical and Electronics Engineering publication 91CH3038-7, p. 216 (1991).

17. H. Brown, J. Cumulat, R.A. Carrigan, C.T. Murphy, S. Peggs, A. Garren, J. Ellison, J. Trischuk, D. Kaplan, B. Newberger, J. Shih, T. Toohig, S. Conetti, B. Cox, and B. Norum, "Report of the fixed target beauty facility working group on progress towards the SFT at the SSC," in Research Directions for the Decade: Proceedings of the 1990 Summer Study on High Energy Physics, E. Berger, ed. (World Scientific, Singapore, 1992) p. 373.

18. B.N. Jensen, S.P. Møller, E. Uggerh øj, T. Worm, K. Elsener, G. Fidecaro, M. Fidecaro, A. Calcaterra, R. DeSangro, I. Peruzzi, E. Gorinin, O. Palamara, S. Petrera, D. Websdale, R. Bellazzini, A. Brez, G. Carboni, F. Costantini, S. Lami, M.M. Massai, M.R. Torquati, A. Capone, D. DePedis, F. Ferroni, S. Morganti, G. Piredda, R. Santacesaria, M. Hage-Ali, P. Siffert, R. Henes, P. Keppler, K. Maier, L. Busso, D. Gamba, A. Grasso, F. Tosello, L. Lanceri, and P. Poropat, "A proposal to test beam extraction by crystal channeling at the SpS: a first step towards an LHC extracted beam," CERN/DRDC 91-25, 15 July 1991.

19. The SFT Collaboration, "An expression of interest in a Super Fixed Target beauty facility (SFT) at the Superconducting Super Collider," SSC Laboratory Report EOI-14, May 25, 1990.

20. M. Harrison and T. Toohig, "A possible reconfiguration of the SSC test beams facility providing $1 \mathrm{TeV}$ and $20 \mathrm{TeV}$ proton targeting and the possibility of high intensity neutrals," in Proceedings of the 1986 Summer Study on the Physics of the Superconducting Supercollider, R. Donaldson and J. Marx, eds., p. 534.

21. Richard A. Carrigan, Jr., "The application of channeling in bent crystals to charged particle beams" in Relativistic Channeling, Richard A. Carrigan, Jr., and James A. Ellison, eds. (Plenum, New York, 1987), p. 339.

22. S.P. Møller, E. Uggerhøj, H.W. Atherton, M. Clément, N. Doble, K. Elsener, L. Gatignon, P. Grafström, M. Hage-Ali, and P. Siffert, "High efficiency bending of $450 \mathrm{GeV}$ protons using channeling," Phys. Lett. B 256, 91 (1991).

23. A.A. Asseev, M.D. Bavizhev, E.A. Ludmirsky, V.A. Maisheev, and Yu. S. Fedotov, "Extraction of the $70 \mathrm{GeV}$ proton beam from the IHEP Accelerator Towards Beam Line 2(14) with a Bent Single Crystal," Nucl. Instrum. Meth., A 309, 1 (1991).

24. R.A. Carrigan, Jr., G. Jackson, C.T. Murphy, S. Peggs, S. Baker, A. Chao, H.-J. Shih, T. Toohig, M. Arenton, S. Conetti, B. Cox, C. Dukes, V. Golovatyk, A. McManus, K. Nelson, B. Norum, A. Boden, D. Cline, J. Rosenzweig, B. Newberger, J.A. Ellison, A. Erwin, R. Rossmanith, A. Sadovsky, A. Taratin, E. Tsyganov, A. Vodopianov, M. Bavizhev, V. Biryukov, A. Khanzadeev and V. Samsonov, "Proposal for a test of low intensity extraction from the tevatron using channeling in a bent crystal," Fermilab Proposal P-853, 22 May 1991.

25. H.-J. Shih, J.A. Ellison, B.S. Newberger, D. Coleman, and J. Ferrell, "RF noise tolerances at the SSC," SSC Laboratory Report SSCL-520, January 1992.

26. D. Coleman and J. Ferrell, personal communication, March 1991.

27. B.S. Newberger, J.A. Ellison, and H.-J. Shih, "Effect of betatron motion on particle loss due to longitudinal diffusion in high-energy colliders," Phys Rev. Lett. 71, 356 (1993).

28. W. Gabella, J. Rosenzweig, R. Kick, and S. Peggs, "RF voltage modulation at discrete frequencies, with application to crystal channeling extraction," Fermilab Report TM-1783, May 1992. 